\title{
LOCALIZATION AND PERIODICITY IN UNSTABLE HOMOTOPY THEORY
}

\author{
A. K. BOUSFIELD
}

\section{INTRODUCTION}

In this paper, we develop a hierarchy of natural localizations of spaces, called the $v_{n}$-periodizations for $n \geq 0$, which may be used to expose and study periodic phenomena in unstable homotopy theory. These $v_{n}$-periodizations act less radically than the corresponding homological localizations [2] and respect fibrations to a very considerable extent. A major part of this paper is devoted to developing the general theory of periodizations of spaces, thereby providing a foundation for the study of the $v_{n}$-periodization and many others. Some of this general theory has been developed independently by Dror Farjoun [13], [14], and we refer the reader to his work for an alternative approach with other interesting general results. During the past decade, remarkable progress was made by Ravenel, Hopkins, Devinatz, and Smith [12], [16], [17], [33] toward a global understanding of stable periodic phenomena, and we hope that the present paper will help to prepare the way for a similar understanding of unstable periodic phenomena.

An excellent exposition of localization and periodicity in stable homotopy theory is now available in Ravenel's book [35]. Major features of stable homotopy are understood "chromatically" as manifestations of more basic periodic phenomena. These phenomena belong to a hierarchy starting with those detected rationally, followed by those detected in classical $K$-theory and in the successive Morava $K$-theories. Most fundamentally, each finite $C W$-spectrum has an intrinsic periodicity given by a " $v_{n}$ self-map" which becomes a selfequivalence after suitable localization.

For simplicity, we describe our results in the pointed homotopy category $\mathrm{Ho}_{0}$ of connected $C W$-complexes. For spaces $W, Y \in H o_{0}$, we call $Y W$-periodic

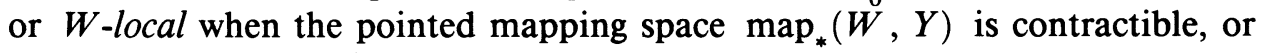
equivalently when $\left[\Sigma^{t} W, Y\right] \cong *$ for $t \geq 0$. As shown more generally in [3, Corollary 7.2], [10], or [13], there is a natural initial example $X \rightarrow P_{W} X$ of a map from $X$ to a $W$-periodic space $P_{W} X$ in $H o_{0}$, and we call this the $W$-periodization or $W$-localization of $X$. Our notation is derived from the classical example where $W=S^{n+1}$ and $P_{W} X$ is the $n$-th Postnikov section

Received by the editors June 18, 1992.

1991 Mathematics Subject Classification. Primary 55P60; Secondary 55N15, 55N20, 55U10.

Research partially supported by the National Science Foundation. 
of $X$. The $v_{n}$-periodization $X \rightarrow P_{v_{n}} X$ will be given by $X \rightarrow P_{\Sigma W_{n}} X$ for a suitable space $W_{n}$ as explained below.

Our first fibration theorem (Theorem 4.1) shows that each homotopy fiber sequence $F \rightarrow X \rightarrow B$ maps naturally to a homotopy fiber sequence $P_{W} F \rightarrow$ $\bar{X} \rightarrow P_{\Sigma W} B$ where the map $X \rightarrow \bar{X}$ may be viewed as a "mixture" of the $W$ periodization and $\Sigma W$-periodization of $X$. For $n \geq 1$ and a prime $p$, we say that a space $W$ satisfies the $n$-supported p-torsion condition when: $\widetilde{H}_{*}(W ; Z)$ is $p$-torsion, $\widetilde{H}_{i}(W ; Z)=0$ for $i<n$, and $H^{n}(W ; Z / p) \neq 0$. For such a $W$, we prove the key result that there is a natural homotopy fiber sequence

$$
K(G, n+1) \rightarrow P_{\Sigma^{2} W} Y \rightarrow P_{\Sigma W} Y
$$

for some $p$-torsion abelian group $G$. This is similar to the fiber sequence relating Postnikov sections of $Y$, and our actual theorem (Theorem 7.2) is stated more generally so as to include such examples. When $W$ satisfies the $n$-supported $p$-torsion condition and when $F \rightarrow X \rightarrow B$ is a homotopy fiber sequence, we deduce that the map from $P_{\Sigma W} F$ to the homotopy fiber of $P_{\Sigma W} X \rightarrow$ $P_{\Sigma W} B$ has homotopy fiber of the form $K(G, n)$ for some $p$-torsion abelian group $G$. Thus $P_{\Sigma W} F \rightarrow P_{\Sigma W} X \rightarrow P_{\Sigma W} B$ is "almost" a homotopy fiber sequence.

We call two spaces $W$ and $W^{\prime} P$-similar when the $W$-periodic spaces are the same as the $W^{\prime}$-periodic spaces, and we write $\langle W\rangle$ for the $P$-similarity class, or $P$-class, of $W$. We also write $\langle W\rangle \leq\left\langle W^{\prime}\right\rangle$ when each $W^{\prime}$-periodic space is $W$-periodic. Dror Farjoun has independently considered such classes. Like the stable classes in [4], the $P$-classes of spaces also have smash and wedge operations. For a space $W$ satisfying the $n$-supported $p$-torsion condition, we prove that

$$
\langle\Sigma W\rangle=\left\langle\Sigma^{k} W\right\rangle \vee\langle K(Z / p, n+1)\rangle
$$

for each $k \geq 1$. This leads to a $P$-classification theorem (Theorem 9.15) showing that two finite $p$-torsion suspension spaces $\Sigma W$ and $\Sigma W^{\prime}$ have $\langle\Sigma W\rangle=$ $\left\langle\Sigma W^{\prime}\right\rangle$ if and only if $\operatorname{conn}(\Sigma W)=\operatorname{conn}\left(\Sigma W^{\prime}\right)$ and type $(\Sigma W)=\operatorname{type}\left(\Sigma W^{\prime}\right)$, where $\operatorname{conn}(\Sigma W)$ denotes the connectivity of $\Sigma W$ and type $(\Sigma W)$ denotes the smallest integer $m$ such that $\widetilde{K}(m)_{*}(\Sigma W) \neq 0$ for the $m$-th Morava $K$-theory $K(m)$. This result suggests that the problem of determining all possible $P$ classes of finite $C W$-complexes may not be totally out of reach.

For each $n \geq 0$, we find a canonical $P$-class $\left\langle\Sigma W_{n}\right\rangle$ whose iterated suspensions give all of the $P$-classes of finite $p$-torsion suspension spaces of type $n+1$. We then define the $v_{n}$-periodization $Y \rightarrow P_{v_{n}} Y$ to be $Y \rightarrow P_{\Sigma W_{n}} Y$. For each space $Y$, there is a natural chromatic tower (10.4)

$$
P_{v_{0}} Y \leftarrow P_{v_{1}} Y \leftarrow P_{v_{2}} Y \leftarrow \cdots
$$

with homotopy inverse limit equivalent to $Y$. This provides successive approximations to $Y$ allowing "successively higher sorts of periodicity".

For a finite $p$-torsion space $V_{n-1}$ of type $n$ with " $v_{n}$ self-map" $\omega: \Sigma^{d} V_{n-1}$ $\rightarrow V_{n-1}$, the $v_{n}$-periodic homotopy groups $v_{n}^{-1} \pi_{*}\left(Y ; V_{n-1}\right)$ may be constructed by inverting the action of $\omega$ on $\pi_{*}\left(Y ; V_{n-1}\right)$. These groups, especially for 
$n=1$ and $n=2$, have figured prominently in recent work of Mahowald, Thompson, and others (see [20], [23], [24]). We show that

$$
\begin{aligned}
& v_{m}^{-1} \pi_{*}\left(P_{v_{n}} Y ; V_{m-1}\right) \cong \begin{cases}v_{m}^{-1} \pi_{*}\left(Y ; V_{m-1}\right) & \text { for } m \leq n, \\
0 & \text { for } m>n,\end{cases} \\
& \pi_{t}\left(P_{v_{n}} Y ; V_{n-1}\right) \cong v_{n}^{-1} \pi_{t}\left(Y ; V_{n-1}\right) \quad \text { for } t \geq 2 \text {. }
\end{aligned}
$$

Thus the $v_{n}$-periodic homotopy groups $v_{n}^{-1} \pi_{*}\left(Y ; V_{n-1}\right)$ are exposed as ordinary homotopy groups of $P_{v_{n}} Y$ with coefficients in $V_{n-1}$.

We establish general homotopical and homological characterizations (13.3 and 13.15) of $P_{v_{n}}$-equivalences for highly connected spaces. These lead, for instance, to a comparison of $v_{1}^{-1} \pi_{*}(-; Z / p)$-equivalences and $K_{*}(-; Z / p)$ equivalences in the pointed homotopy category $\mathrm{Ho}_{3}$ of 3-connected spaces. A map $\varphi: X \rightarrow Y$ in $\mathrm{Ho}_{3}$ is called a durable $K_{*}(-; Z / p)$-equivalence when $\widetilde{\Omega}^{k} \varphi: \widetilde{\Omega}^{k} X \rightarrow \widetilde{\Omega}^{k} Y$ is a $K_{*}(-; Z / p)$-equivalence for each $k \geq 0$, where $\widetilde{\Omega}$ : $\mathrm{Ho}_{3} \rightarrow \mathrm{Ho}_{3}$ carries each space to the 3-connected cover of its loop space. In 14.7, we prove that the following conditions on a map $\varphi: X \rightarrow Y$ in $\mathrm{Ho}_{3}$ are equivalent:

(i) $\varphi$ is a $v_{1}^{-1} \pi_{*}(-; Z / p)$-equivalence;

(ii) $\varphi$ is a durable $K_{*}(-; Z / p)$-equivalence;

(iii) $\widetilde{\Omega}^{k} \varphi$ is a $K_{*}(-; Z / p)$-equivalence for some $k \geq 2$.

This is an unstable version of the result, proved in [5] using work of Mahowald and Miller, that a map $\theta: E \rightarrow F$ of spectra is a $v_{1}^{-1} \pi_{*}(-; Z / p)$-equivalence if and only if it is a $K_{*}(-; Z / p)$-equivalence. As an application, we verify the old conjecture of Miller-Snaith [26] and Mahowald-Ravenel [21] that the Snaith map

$$
s: \Omega_{0}^{2 n+1} S^{2 n+1} \rightarrow Q\left(\mathbb{R} P^{2 n}\right)
$$

is a $K_{*}(-; Z / 2)$-equivalence for $n \geq 1$. This is now an immediate corollary of Mahowald's theorem [20] that the Snaith map is a $v_{1}^{-1} \pi_{*}(-; Z / 2)$-equivalence. Since the algebra $K_{*}\left(Q\left(\mathbb{R} P^{2 n}\right) ; Z / 2\right)$ is known from work of Miller-Snaith [27], the hitherto inaccessible algebra $K_{*}\left(\Omega_{0}^{2 n+1} S^{2 n+1} ; Z / 2\right)$ is now also known. In very recent work, Lisa Langsetmo [19] has similarly determined all of the algebras $K_{*}\left(\Omega^{j} S^{2 n+1} ; Z / p\right)$ for $j<2 n$ using $v_{1}^{-1} \pi_{*}(-; Z / p)$-equivalences derived from the work of Mahowald-Thompson [24] together with $K$-theoretic calculations on the resulting "infinite loop space related" models for $\Omega^{j} S^{2 n+1}$. We believe that our results should permit other $K$-theoretic calculations of this sort.

This paper initially grew from our efforts to understand work of Mahowald and Thompson from a more general perspective. We also wish to thank Dror Farjoun for a useful exchange of preliminary manuscripts in 1991 after we had independently developed our main results in this area.

This paper is written simplicially so that "space" means "simplicial set". However, for convenience, many results are presented in the pointed homo- 
topy category $\mathrm{Ho}_{*}$, whose objects may be taken as pointed $\mathrm{CW}$-complexes when desired.

\section{THE PERIODIZATION OF SPACES}

For pointed spaces $A, Y$, let $\operatorname{map}_{*}(A, Y)$ and $\operatorname{map}(A, Y)$ respectively denote the pointed and unpointed mapping spaces, and recall that $\pi_{0} \operatorname{map}_{*}(A, Y)$ $\cong[A, Y]$ when $Y$ is fibrant. For a pointed map $f: A \rightarrow B$ and fibrant pointed space $Y$, consider the conditions:
(H1) $f^{*}:[B, Y] \cong[A, Y]$;
(H2) $f^{*}: \operatorname{map}_{*}(B, Y) \simeq \operatorname{map}_{*}(A, Y)$;
(H3) $f^{*}: \operatorname{map}(B, Y) \simeq \operatorname{map}(A, Y)$.

Lemma 2.1. In general, $(\mathrm{H} 3) \Rightarrow(\mathrm{H} 2) \Rightarrow(\mathrm{H} 1)$. When $Y$ is connected, $(\mathrm{H} 2)$ $\Leftrightarrow(\mathrm{H} 3)$.

Proof. This follows using the natural fiber sequence

$$
\operatorname{map}_{*}(A, Y) \rightarrow \operatorname{map}(A, Y) \rightarrow Y .
$$

The mapping space functors may be applied in the pointed homotopy category $\mathrm{Ho}_{*}$ using fibrant versions of target spaces, and we adopt (H3) as our main "orthogonality" condition in $H o_{*}$. For spaces $W, Y \in H o_{*}$, we call $Y$ $W$-periodic or $W$-local when $W \rightarrow *$ induces an equivalence $Y \simeq \operatorname{map}(W, Y)$. Thus, for $W$ connected, $Y$ is $W$-periodic if and only if $\operatorname{map}_{*}\left(W, Y_{\alpha}\right) \simeq *$ for each component $Y_{\alpha}$ of $Y$, where an arbitrary basepoint is chosen in $Y_{\alpha}$. A map $f: A \rightarrow B$ in $\stackrel{\alpha}{H} o_{*}$ will be called a $W$-periodic equivalence or $W$-local equivalalence when $f^{*}: \operatorname{map}(B, Y) \simeq \operatorname{map}(A, Y)$ for each $W$-periodic space $Y \in H o_{*}$. Thus, for $W$ connected, a map $f: A \rightarrow B$ is a $W$-periodic equivalence if and only if $f_{*}: \pi_{0} A \cong \pi_{0} B$ and $f_{\alpha}^{*}: \operatorname{map}_{*}\left(B_{\alpha}, Y\right) \simeq \operatorname{map}_{*}\left(A_{\alpha}, Y\right)$ for each connected $W$-periodic space $Y \in H o_{*}$ and for each submap $f_{\alpha}: A_{\alpha} \rightarrow B_{\alpha}$ of components, where compatible basepoints are chosen in $A_{\alpha}$ and $B_{\alpha}$. Note that $W \rightarrow *$ is itself a $W$-periodic equivalence. A map $u: X \rightarrow Y$ in $H o_{*}$ will be called a $W$-periodization or $W$-localization of $X$ when $u$ is a $W$-periodic equivalence and $Y$ is $W$-periodic. The localization theorems of the author [3, Corollary 7.2], Casacuberta-Peschke-Pfenniger [10], or Dror Farjoun [13] specialize to give

Theorem 2.2. For each $W, X \in H o_{*}$, there exists a $W$-periodization of $X$.

This periodization is clearly unique up to equivalence and will be denoted by $u: X \rightarrow P_{W} X$. It is the initial example of a map from $X$ to a $W$-periodic space in $\mathrm{Ho}_{*}$, and also the terminal example of a $W$-periodic equivalence out of $X$ in $\mathrm{Ho}_{*}$. General constructions of $P_{W} X$ will be briefly discussed in 2.8 and 2.10 .

Example 2.3. If $W$ is a point, then $P_{W} X \simeq X$ for each $X \in H o_{*}$. If $W$ is not connected or if $X=W$, then $P_{W} X \simeq *$.

Example 2.4. If $W=S^{n+1}$ for $n \geq 0$, then $u: X \rightarrow P_{W} X$ is the $n$-th Postnikov section for each $X \in H o_{*}$. 
The reader should keep this Postnikov example in mind, since many of its formal properties will generalize.

For $W, X \in H o_{*}$ we have

$$
u \simeq P_{W} u: P_{W} X \simeq P_{W} P_{W} X,
$$

and we may therefore view $P_{W}: H o_{*} \rightarrow H o_{*}$ and $u$ : Ident $\rightarrow P_{W}$ as an idempotent functor on $\mathrm{Ho}_{*}$. We shall use the term $P_{W}$-equivalence as a synonym for $W$-periodic equivalence.

2.5. Closure properties of $P_{W}$-equivalences. For $W \in H o_{*}$ and a map $f: A \rightarrow$ $B$ of pointed spaces, the following conditions are equivalent:

(a) $f$ is a $P_{W}$-equivalence;

(b) $f^{*}:[B, Y] \cong[A, Y]$ for each $W$-periodic space $Y \in H o_{*}$;

(c) $f^{*}: \operatorname{map}_{*}(B, Y) \simeq \operatorname{map}_{*}(A, Y)$ for each $W$-periodic space $Y \in H o_{*}$;

(d) $f^{*}: \operatorname{map}(B, Y) \simeq \operatorname{map}(A, Y)$ for each $W$-periodic space $Y \in H o_{*}$.

Consequently, the $P_{W}$-equivalences are closed under homotopy colimits in both the pointed and unpointed senses. Some basic cases are:

(i) for a set $\left\{A_{\alpha} \rightarrow B_{\alpha}\right\}$ of $P_{W}$-equivalences, the wedge $\vee_{\alpha} A_{\alpha} \rightarrow \vee_{\alpha} B_{\alpha}$ is a $P_{W}$-equivalence;

(ii) for a pointed space $J$ and $P_{W}$-equivalence $A \rightarrow B$, the maps $J \wedge B \rightarrow$ $J \wedge B$ and $J \times A \rightarrow J \times B$ are $P_{W}$-equivalences;

(iii) for a homotopy cofiber square

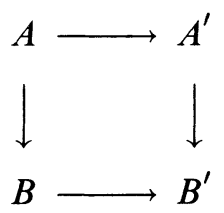

of pointed spaces, if $A \rightarrow B$ is a $P_{W}$-equivalence then so is $A^{\prime} \rightarrow B^{\prime}$;

(iv) for a possibly transfinite sequence of pointed spaces $A_{0} \rightarrow A_{1} \rightarrow \cdots$, if each $A_{0} \rightarrow A_{\alpha}$ is a $P_{W}$-equivalence, then so is $A_{0} \rightarrow \underset{\alpha}{\operatorname{hocolim}} A_{\alpha}$.

2.6. Closure properties of $W$-periodic spaces. For $W \in H o_{*}$, the fibrant $W$ periodic pointed spaces are closed under homotopy limits. Some basic examples are:

(i) for a set $\left\{Y_{\alpha}\right\}$ of fibrant $W$-periodic pointed spaces, the product $\prod_{\alpha} Y_{\alpha}$ is $W$-periodic;

(ii) for a pointed space $J$ and fibrant $W$-periodic pointed space $Y$, the mapping spaces $\operatorname{map}_{*}(J, Y)$ and $\operatorname{map}(J, Y)$ are $W$-periodic;

(iii) for a homotopy fiber square

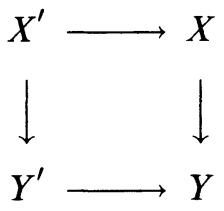

of pointed spaces, if $X, Y, Y^{\prime}$ are $W$-periodic, then so is $X^{\prime}$; 
(iv) for a possibly transfinite tower $A_{0} \leftarrow A_{1} \leftarrow \cdots$ of fibrant pointed spaces, if each $A_{\alpha}$ is $W$-periodic, then so is $\underset{\alpha}{\lim } A_{\alpha}$.

An immediate consequence of 2.5 (ii) and 2.6 (i) is

Proposition 2.7. For $W \in H o_{*}$ the natural map

$$
P_{W}\left(X_{1} \times \cdots \times X_{n}\right) \rightarrow P_{W}\left(X_{1}\right) \times \cdots \times P_{W}\left(X_{n}\right)
$$

is a homotopy equivalence for each finite set of spaces $X_{1}, \ldots, X_{n} \in H o_{*}$.

We briefly describe two general constructions of $P_{W} X$.

2.8. A topological construction of $P_{W} X$. For connected $C W$-complexes $W, X$ $\in H o_{*}$, let $\lambda$ be the first infinite ordinal with cardinality greater than the number of cells in $W$, and inductively construct an increasing sequence of $C W$ complexes

$$
X=X(0) \subset X(1) \subset \cdots \subset X(\alpha) \subset X(\alpha+1) \subset \cdots \subset X(\lambda)
$$

indexed by the ordinals $\leq \lambda$ as follows. Given $X(\alpha)$, choose a set of pointed cellular maps $\left\{g: \Sigma^{i} W \rightarrow X(\alpha)\right\}_{g \in G(i)}$ for each $i \geq 0$ representing all the pointed homotopy classes from $\Sigma^{i} W$ to $X(\alpha)$, and let $X(\alpha+1)$ be the mapping cone of the induced map

$$
\bigvee_{i \geq 0} \bigvee_{g \in G(i)} \Sigma^{i} W \rightarrow X(\alpha)
$$

Also, let $X(\beta)=\bigcup_{\alpha<\beta} X(\alpha)$ for each limit ordinal $\beta$. The map $X \rightarrow X(\lambda)$ produced by induction is a $W$-periodization, and thus $P_{W} X \simeq X(\lambda)$. An easy consequence of this construction is

Proposition 2.9. For $W, X \in H o_{*}$ with $W$ n-connected, $u_{*}: \pi_{i} X \rightarrow \pi_{i} P_{W} X$ is bijective for $i \leq n$ and onto for $i=n+1$.

Our second general construction of $P_{W} X$ involves a functor

$$
P_{W}: \text { Spaces } \rightarrow \text { Spaces }
$$

on the category of unpointed spaces (i.e., simplicial sets). We use a construction from [6] since it provides a simplicial functor in the sense of Quillen [32, II, §1], whereas other available functors on simplicial sets or topological spaces seem to lack this continuity property. For $W, Y \in$ Spaces with $Y$ fibrant, we call $Y W$-periodic when $W \rightarrow *$ induces an equivalence $Y \simeq \operatorname{map}(W, Y)$.

Theorem 2.10. For each space $W$, there exists a functor $P_{W}:$ Spaces $\rightarrow$ Spaces together with natural maps $u: X \subset P_{W} X$ and $c: A \times P_{W} X \rightarrow P_{W}(A \times X)$ such that for all spaces $A, B, X$ the following hold:

(i) $P_{W} X$ is a fibrant $W$-periodic space;

(ii) $u^{*}: \operatorname{map}\left(P_{W} X, Y\right) \simeq \operatorname{map}(X, Y)$ for each fibrant $W$-periodic space $Y$

(iii) $P_{W}(*)=*$; 
(iv) the canonical map

$$
P_{W} X \cong * \times P_{W} X \stackrel{\mathcal{c}}{\rightarrow} P_{W}(* \times X) \cong P_{W} X
$$

is the identity;

(v) there are equalities

$$
\begin{aligned}
& c=u(A \times c): A \times B \times P_{W} X \rightarrow P_{W}(A \times B \times X), \\
& u=c(B \times u): B \times X \rightarrow P_{W}(B \times X) .
\end{aligned}
$$

Proof. Let $\left\{A_{\alpha} \subset B_{\alpha}\right\}$ consist of the inclusions

$$
\begin{gathered}
\left(W \times \Delta^{n}\right) \cup\left(C W \times \dot{\Delta}^{n}\right) \\
V_{i}^{n+1} \subset C W \times \Delta^{n+1},
\end{gathered}
$$

for all $n \geq 0$ and $0 \leq i \leq n+1$, where $\dot{\Delta}^{n}$ is the boundary of the standard $n$-simplex $\Delta^{n}$ and $V_{i}^{n+1}$ is the union of all faces of $\Delta^{n+1}$ except for the $i$-th. The construction of [6] with respect to $\left\{A_{\alpha} \subset B_{\alpha}\right\}$ now gives the desired functor and transformations.

Note. For a pointed space $X$, the above inclusion $u: X \subset P_{W} X$ represents the $W$-periodization of $X$ in $\mathrm{Ho}_{*}$, and thus $P_{W}:$ Spaces $\rightarrow$ Spaces induces $P_{W}$ : $\mathrm{Ho}_{*} \rightarrow \mathrm{Ho}_{*}$.

2.11. A generalization. By [3, Corollary 7.2], [10], or [13], the elementary theory of $W$-periodizations can be generalized to a theory of $f$-periodizations (equivalently called $f$-localizations) using an arbitrary map $f: W \rightarrow W^{\prime}$ of spaces in place of $W \rightarrow *$. Thus, a space $Y \in H_{*}$ is called $f$-periodic or $f$-local when $f^{*}: \operatorname{map}\left(W^{\prime}, Y\right) \simeq \operatorname{map}(W, Y)$; a map $\varphi: A \rightarrow B$ in $H o_{*}$ is called an $f$-periodic equivalence or $f$-local equivalence when $\varphi^{*}: \operatorname{map}(B, Y) \simeq$ $\operatorname{map}(A, Y)$ for each $f$-periodic space $Y \in H o_{*}$; a map $u: X \rightarrow Y$ in $H o_{*}$ is called an $f$-periodization or $f$-localization when it is an $f$-periodic equivalence and $Y$ is $f$-periodic. The terminology and results of 2.2, 2.5, 2.6, 2.7, and 2.10 all apply immediately with $f$ in place of $W$.

\section{The equivalence of $P_{W}(\Omega Y)$ and $\Omega\left(P_{\Sigma W} Y\right)$}

For $W, Y \in H o_{*}$, the space $\Omega\left(P_{\Sigma W} Y\right) \in H o_{*}$ is $W$-periodic, and thus there is a unique map $\lambda: P_{W}(\Omega Y) \rightarrow \Omega\left(P_{\Sigma W} Y\right)$ in $H o_{*}$ such that

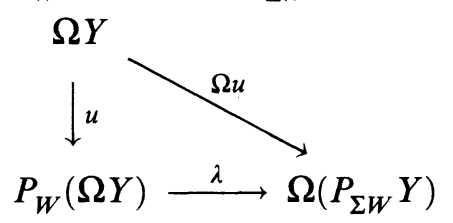

commutes. In this section, we shall prove that $\lambda$ is an equivalence by destabilizing work of [6] on the localization of infinite loop spaces. The following theorem and corollary were obtained independently by Dror Farjoun [14].

Theorem 3.1. For $W, Y \in H o_{*}$, the natural map $\lambda: P_{W}(\Omega Y) \rightarrow \Omega\left(P_{\Sigma W} Y\right)$ is a homotopy equivalence.

This may be applied repeatedly to give 
Corollary 3.2. For $W, Y \in H o_{*}$ and $k \geq 1$, the natural map $\lambda^{k}: P_{W}\left(\Omega^{k} Y\right) \rightarrow$ $\Omega^{k}\left(P_{\Sigma^{k} W} Y\right)$ is a homotopy equivalence.

For the proof of 3.1 and for later use, we need

3.3. Segal's theory of loop spaces. In preparation for his work on infinite loop spaces, Segal [37] introduced a theory of single loop spaces. The following version will be developed more generally for iterated loop spaces in [7]. For $m \geq 0$, let $\Delta^{m} / \mathrm{sk}^{0} \Delta^{m}$ be the space formed by pinching the 0 -skeleton of $\Delta^{m}$ to a point, and for a pointed space $Y$, let $\Omega_{\text {bis }} Y$ be the pointed bisimplicial set with

$$
\left(\Omega_{\text {bis }} Y\right)_{m, \bullet}=\operatorname{map}_{*}\left(\Delta^{m} / \text { sk }^{0} \Delta^{m}, Y\right) \text {. }
$$

Note that $\left(\Omega_{\text {bis }} Y\right)_{0, \bullet}=*$ and $\left(\Omega_{\text {bis }} Y\right)_{1, \bullet}=\Omega Y$. A bisimplicial set $X$ is called horizontally reduced when $X_{0, \bullet}=*$, and is called very special when it is horizontally reduced and

$$
\rho_{1} \times \cdots \times \rho_{m}: X_{m, \bullet} \rightarrow X_{1, \bullet} \times \cdots \times X_{1, \bullet}
$$

is a weak equivalence for each $m \geq 1$, where $\rho_{i}: X_{m, \bullet} \rightarrow X_{1, \bullet}$ is the horizontal simplicial operator corresponding to the monotone function $\rho_{i}:\{0,1\} \rightarrow$ $\{0,1, \ldots, m\}$ with $\rho_{i}(0)=0$ and $\rho_{i}(1)=i$. This is equivalent to the condition that $X$ is special in Segal's sense with $\pi_{0} X_{1, \bullet}$ a group. With our choice of operators, we do not need a separate condition on $\pi_{0} X_{1, \bullet}$. When $Y$ is fibrant, $\Omega_{\text {bis }} Y$ is very special and "captures the higher multiplicative structure of $\Omega Y$ ". The functor $\Omega_{\text {bis }}$ from pointed spaces to horizontally reduced bisimplicial sets is right adjoint to the diagonal functor diag. Using [8, Appendix B], as explained more generally in [7], one shows:

(i) for a pointed connected fibrant space $Y$, the adjunction counit $\operatorname{diag} \Omega_{\text {bis }} Y \rightarrow Y$ is a weak equivalence;

(ii) for a very special bisimplicial set $X$, the natural map $X_{1, \bullet} \rightarrow$ $\Omega \mathrm{Ex}^{\infty} \operatorname{diag} X$ is a weak equivalence, where Kan's [18] functor $\mathrm{Ex}^{\infty}$ is used to "make $\operatorname{diag} X$ fibrant".

3.4. The classifying space of $P_{W}(\Omega Y)$. For a pointed connected fibrant space $Y$, we consider the pointed space $T_{W} Y=\operatorname{diag} P_{W} \Omega_{\text {bis }} Y$ and the natural maps

$$
Y \cong \operatorname{diag} \Omega_{\mathrm{bis}} Y \rightarrow \operatorname{diag} P_{W} \Omega_{\mathrm{bis}} Y=T_{W} Y
$$

obtained from 2.10 and 3.3 (i). These determine a functor $T_{W}: \mathrm{Ho}_{0} \rightarrow \mathrm{Ho}_{0}$ with coaugmentation $\bar{u}$ : Ident $\rightarrow T_{W}$, where $H o_{0} \subset H o_{*}$ is the full subcategory of connected spaces. By 2.7 the bisimplicial set $P_{W} \Omega_{\text {bis }} Y$ is very special, and thus by 3.3 (ii) there is a natural equivalence $\beta: P_{W} \Omega Y \simeq \Omega T_{W} Y$ with $\beta u$ $=\Omega \bar{u}: \Omega Y \rightarrow \Omega T_{W} Y$ for $Y \in H o_{0}$. Consequently, $T_{W} Y$ is the classifying space of $P_{W} \Omega Y$ and:

(i) a space $Y \in H o_{0}$ has $\bar{u}: Y \simeq T_{W} Y$ if and only if $\Omega Y$ is $W$-periodic in $\mathrm{Ho}_{*}$;

(ii) a map $\varphi: X \rightarrow Y$ in $H o_{0}$ has $T_{W} \varphi: T_{W} X \simeq T_{W} Y$ if and only if $\Omega \varphi: \Omega X \rightarrow \Omega Y$ is a $P_{W}$-equivalence in $H_{*}$. 
Thus, the maps $\bar{u}, T \bar{u}: T_{W} Y \rightarrow T_{W} T_{W} Y$ are equivalences for each $Y \in H o_{0}$ and must be equal by [6, Lemma 6.6]. In other words, $T_{W}: H o_{0} \rightarrow H o_{0}$ and $\bar{u}:$ Ident $\rightarrow T_{W}$ constitute an idempotent functor on $\mathrm{Ho}_{0}$.

3.5. Proof of Theorem 3.1. Since $P_{W} \Omega Y$ and $\Omega P_{\Sigma W} Y$ depend only on the base component of $Y$, we may assume that $Y$ is connected. The idempotent functors $T_{W}$ and $P_{\Sigma W}$ on $\mathrm{Ho}_{0}$ must be equivalent since they have the same local spaces, namely those with $W$-periodic loop spaces. Thus there is a natural equivalence $\bar{\lambda}: T_{W} Y \simeq P_{\Sigma W} Y$ such that the triangle

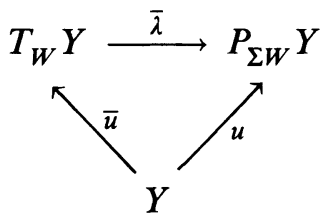

commutes. Applying $\Omega$, we find that $\lambda: P_{W} \Omega Y \rightarrow \Omega P_{\Sigma W} Y$ is an equivalence since it is the composite of the equivalences $\beta: P_{W} \Omega Y \simeq \Omega T_{W} Y$ and $\Omega \bar{\lambda}$ : $\Omega T_{W} Y \simeq \Omega P_{\Sigma W} Y$.

3.6. A generalization. We note that the terminology and results of 3.1, 3.2, and 3.4 all apply immediately when a map of spaces is used in place of $W$ as in 2.11 .

\section{A GENERAL FIBRATION THEOREM}

In this section, we prove the following general fibration theorem and explore some of its consequences. It will be used in Section 8 to prove our main fibration theorem.

Theorem 4.1. For a pointed space $W$ and a homotopy fiber sequence $F \rightarrow X \rightarrow$ $B$ of pointed spaces with $B$ connected, there is a natural homotopy fiber sequence $P_{W} F \rightarrow \bar{X} \rightarrow P_{\Sigma W} B$ such that $\bar{X}$ is $\Sigma W$-periodic. Moreover, there is a natural map

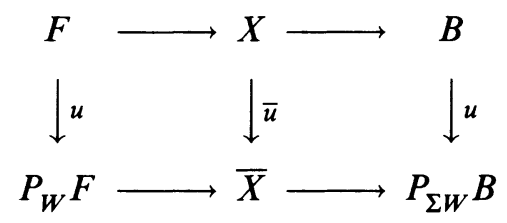

in the homotopy category of homotopy fiber sequences where $\bar{u}: X \rightarrow \bar{X}$ is a $P_{W}$-equivalence.

This will be proved in 4.10. We view $\bar{u}: X \rightarrow \bar{X}$ as a "mixture" of the $W$-periodization and $\Sigma W$-periodization of $X$.

Proposition 4.2. For a pointed space $W$ and homotopy fiber sequence $F \rightarrow X \rightarrow$ $B$ with $B$ connected, the following hold:

(i) if $F$ and $B$ are $W$-periodic, then so is $X$;

(ii) if $F$ is $W$-periodic and $X$ is $\Sigma W$-periodic, then $B$ is $\Sigma W$-periodic;

(iii) if $X$ is $W$-periodic and $B$ is $\Sigma W$-periodic, then $F$ is $W$-periodic.

This is easily proved, and Theorem 4.1 now implies 
Theorem 4.3. For a pointed space $W$ and pointed connected space $B$ with $P_{\Sigma W} B \simeq P_{W} B$, the functor $P_{W}$ carries each homotopy fiber sequence $F \rightarrow X \rightarrow$ $B$ to a homotopy fiber sequence $P_{W} F \rightarrow P_{W} X \rightarrow P_{W} B$.

This is due to Dror Farjoun [14] when $B$ is $W$-periodic. For a pointed space $X$, let $X\langle n\rangle \rightarrow X$ be the $n$-connected cover, and let $X \rightarrow P^{n} X$ be the $n$-th Postnikov section.

Corollary 4.4. For a pointed $n$-connected space $W$ with $n \geq 0$ and pointed connected space $X$, there are natural equivalences

$$
\begin{aligned}
P_{W}(X\langle n\rangle) & \simeq\left(P_{W} X\right)\langle n\rangle, \\
P^{n} X & \simeq P^{n}\left(P_{W} X\right) .
\end{aligned}
$$

Proof. Apply 4.3 to the homotopy fiber sequence $X\langle n\rangle \rightarrow X \rightarrow P^{n} X$ and note that $P_{W}(X\langle n\rangle)$ is $n$-connected by 2.9 .

4.5. Fiberwise localizations. For a pointed space $W$ and a homotopy fiber sequence $F \rightarrow X \rightarrow B$ of pointed spaces with $B$ connected, there is a natural homotopy fiber sequence $P_{W} F \rightarrow X^{\prime} \rightarrow B$ and a natural map

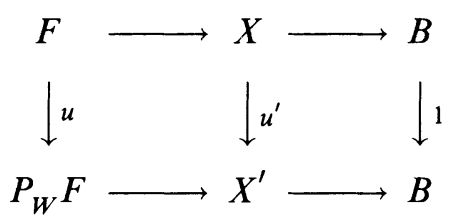

in the homotopy category of homotopy fiber sequences obtained by pulling back the map of 4.1. The map $u^{\prime}: X \rightarrow X^{\prime}$ is a $P_{W}$-equivalence by 4.7 below.

The following theorem extends a result of Zabrodsky and Miller [25, Proposition 9.5] and will be proved in 4.11.

Theorem 4.6. Let

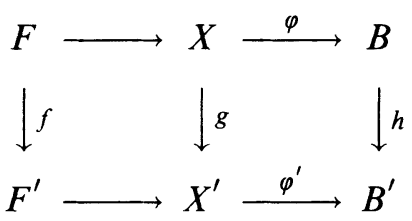

be a map of homotopy fiber sequences with $B$ and $B^{\prime}$ connected. For a fibrant space $Y$, if $f^{*}: \operatorname{map}\left(F^{\prime}, Y\right) \simeq \operatorname{map}(F, Y)$ and $(\Omega h)^{*}: \operatorname{map}\left(\Omega B^{\prime}, Y\right) \simeq$ $\operatorname{map}(\Omega B, Y)$, then $g^{*}: \operatorname{map}\left(X^{\prime}, Y\right) \simeq \operatorname{map}(X, Y)$. When $Y$ is pointed and connected, "map" may be replaced by "map ${ }_{*}$ ". For a spectrum $E$, if $f: F \rightarrow F^{\prime}$ and $\Omega h: \Omega B \rightarrow \Omega B^{\prime}$ are $E^{*}$-equivalences or $E_{*}$-equivalences, then so is $g$ : $X \rightarrow X^{\prime}$.

Corollary 4.7. For a pointed space $W$ and map

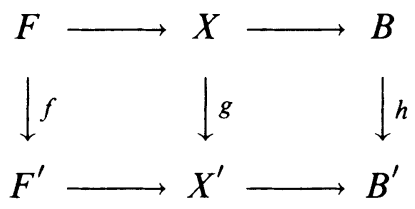


of homotopy fiber sequences with $B$ and $B^{\prime}$ connected, the following hold:

(i) if $f$ is a $P_{W}$-equivalence and $h$ is a $P_{\Sigma W^{-}}$equivalence, then $g$ is a $P_{W}$-equivalence;

(ii) if $g$ and $h$ are $P_{\Sigma W}$-equivalences, then $f$ is a $P_{W}$-equivalence;

(iii) if $f$ and $g$ are $P_{\Sigma W^{-}}$-quivalences, then $h$ is a $P_{\Sigma W^{-e q u i v a l e n c e .}}$

Proof. Part (i) follows from 4.6, and the other parts follow from (i) using 3.1.

Corollary 4.7 specializes to give

Corollary 4.8. For a pointed space $W$ and homotopy fiber sequence $F \rightarrow X \rightarrow B$ with $B$ connected, the following hold:

(i) if $P_{W} F \simeq *$, then $X \rightarrow B$ is a $P_{W}$-equivalence;

(ii) if $X \rightarrow B$ is a $P_{\Sigma W}$-equivalence, then $P_{W} F \simeq *$;

(iii) if $P_{\Sigma W} B \simeq *$, then $F \rightarrow X$ is a $P_{W}$-equivalence;

(iv) if $F \rightarrow X$ is a $P_{\Sigma W}$-equivalence, then $P_{\Sigma W} B \simeq *$.

We devote the rest of this section to proving 4.1 and 4.6. Let $\varphi: X \rightarrow B$ be a fibration of pointed spaces with $B$ connected and fibrant, and let $F$ be the fiber of $\varphi$. The termwise cofiber sequence of pairs

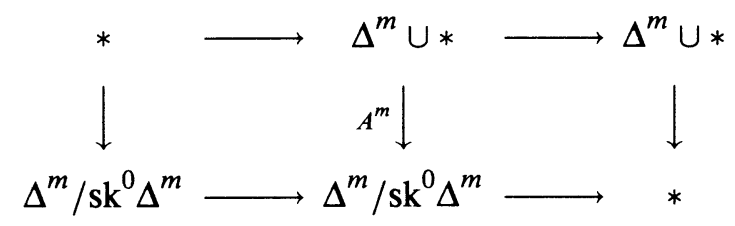

for $m \geq 0$ is carried by $\operatorname{map}_{*}(-, \varphi)$ to a fiber sequence

$$
\operatorname{map}\left(\Delta^{m}, F\right) \rightarrow \operatorname{map}_{*}\left(A^{m}, \varphi\right) \rightarrow \operatorname{map}_{*}\left(\Delta^{m} / \mathbf{s k}^{0} \Delta^{m}, B\right)
$$

of pointed spaces, and this is homotopically equivalent to a projection fiber sequence

$$
F \rightarrow \Omega B \times \cdots \times \Omega B \times F \rightarrow \Omega B \times \cdots \times \Omega B .
$$

Letting $m$ vary, we obtain a termwise fiber sequence of bisimplicial sets

$$
\operatorname{map}\left(\Delta^{\bullet}, F\right) \longrightarrow \operatorname{map}_{*}\left(A^{\bullet}, \varphi\right) \longrightarrow \Omega_{\text {bis }} B .
$$

A more familiar, but less natural, version of this sequence may be obtained by "turning the action of $\Omega B$ on $F$ into a group action and applying the geometric bar construction". For a pointed space $W$, we apply the transformation $u$ : Ident $\rightarrow P_{W}$ termwise and then take diagonals to give a diagram of pointed spaces

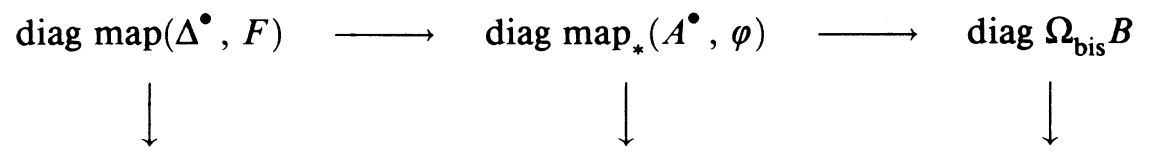

$\operatorname{diag} P_{W} \operatorname{map}\left(\Delta^{\bullet}, F\right) \longrightarrow \operatorname{diag} P_{W} \operatorname{map}_{*}\left(A^{\bullet}, \varphi\right) \longrightarrow \operatorname{diag} P_{W} \Omega_{\text {bis }} B$.

Lemma 4.9. The rows in the above diagram are homotopy fiber sequences.

Proof. This follows by Theorem B.4 of [8] whose hypotheses are straightforward to verify since $P_{W}$ preserves finite products up to homotopy. In particular, 
$\Omega_{\text {bis }} B$ and $P_{W} \Omega_{\text {bis }} B$ satisfy the $\pi_{*}-$ Kan condition by [7] since they are very special.

4.10. Proof of Theorem 4.1. In the natural commutative diagram

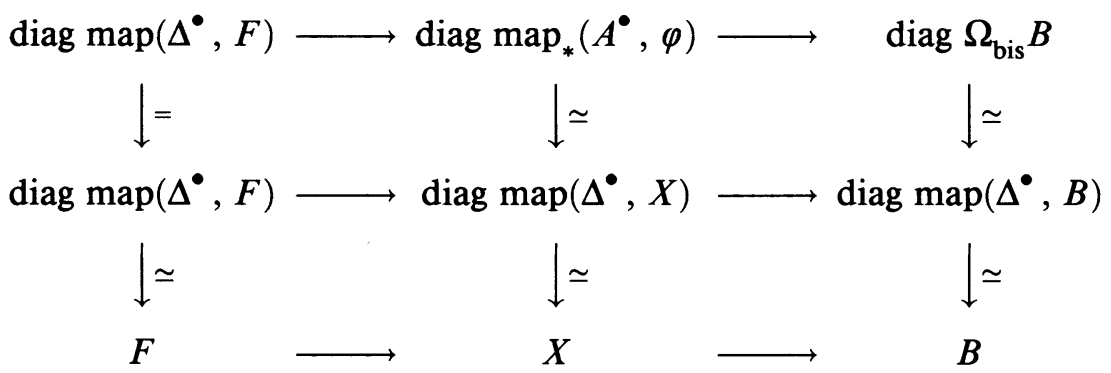

the lower vertical maps are weak equivalences since their canonical right inverses are weak equivalences by [8, B.2], and thus all three rows are homotopy fiber sequences. The composite map diag $\Omega_{\text {bis }} B \rightarrow B$ is the adjunction counit which is a weak equivalence by 3.3 (i). Thus all vertical maps are weak equivalences. Using 3.4 and 3.5 , we conclude that the diagram preceding 4.9 is equivalent to a natural map

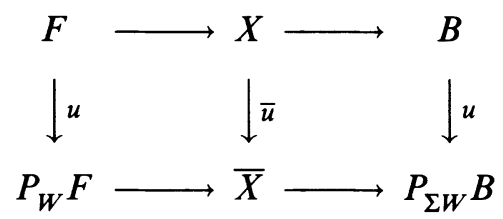

in the homotopy category of homotopy fiber sequences. Since $\bar{X}$ is $\Sigma W$ periodic by 4.2 , and $\bar{u}: X \rightarrow \bar{X}$ is a $P_{W}$-equivalence by 4.7 (i), it remains to give

4.11. Proof of Theorem 4.6. Since the vertical maps in 4.6 induce the equivalences $\operatorname{map}\left(F^{\prime}, Y\right) \simeq \operatorname{map}(F, Y)$ and $\operatorname{map}\left(\Omega B^{\prime}, Y\right) \simeq \operatorname{map}(\Omega B, Y)$, they must also induce the equivalences

$$
\operatorname{map}\left(\operatorname{map}_{*}\left(A^{m}, \varphi^{\prime}\right), Y\right) \simeq \operatorname{map}\left(\operatorname{map}_{*}\left(A^{m}, \varphi\right), Y\right)
$$

for $m \geq 0$. Since $\operatorname{diag}\left(\operatorname{map}_{*}\left(A^{\bullet}, \varphi\right)\right) \simeq X$ by the proof of Theorem 4.1, and since $\operatorname{diag}\left(\operatorname{map}_{*}\left(A^{\bullet}, \varphi\right)\right)$ is equivalent to the homotopy colimit of $\operatorname{map}_{*}\left(A^{\bullet}, \varphi\right)$ by $\left[9\right.$, p. 335], we conclude that $g^{*}: \operatorname{map}\left(X^{\prime}, Y\right) \simeq \operatorname{map}(X, Y)$. The statements for map $_{*}$ and $E^{*}$ now follow by 2.1 , and imply the statement for $E_{*}$ since the $E_{*}$-equivalences are the same as the $(\nabla E)^{*}$-equivalences, where $\nabla E$ is the "Anderson-Yosimura dual" of $E$ with universal coefficient sequence

$$
0 \rightarrow \operatorname{Ext}\left(E_{*-1} X, Z\right) \rightarrow(\nabla E)^{*} X \rightarrow \operatorname{Hom}\left(E_{*} X, Z\right) \rightarrow 0
$$

as in [40]. Alternatively, the statements for $E^{*}$ and $E_{*}$ follow by easy spectral sequence arguments.

4.12. A generalization. When a map of spaces is used in place of $W$ as in 2.11 and 3.6, we no longer have the results of 4.2 on periodicity properties of spaces in a fibration. However, 4.1 still holds without mention of a periodicity 
property for $\bar{X}$. Moreover, the results and proofs of 4.5, 4.7, 4.8, and 4.9 all apply immediately.

\section{Periodizations With Respect to Moore sPaces}

Before continuing with the theory of $W$-periodizations, we consider the case where $W$ is a Moore space or a wedge of Moore spaces. Our results will involve

5.1. The abelian group $A / / G$. For abelian groups $G$ and $X$, we call $X G$ reduced when $\operatorname{Hom}(G, X)=0$. Each abelian group $A$ has a maximal $G$ reduced quotient group denoted by $A / / G$, which may be constructed as the image of the homomorphism from $A$ to the product of all its $G$-reduced quotient groups. The following general examples are easily verified:

(i) for a set $I$ of primes, if $G$ is an $I$-torsion abelian group with $G / p G \neq 0$ for each $p \in I$, then $A / / G$ is the quotient of $A$ by its maximal $I$ torsion subgroup;

(ii) for a set $J$ of primes, if $G$ is a uniquely $J$-divisible abelian group with $\operatorname{Hom}\left(G, Z\left[J^{-1}\right]\right) \neq 0$ (or equivalently with $G$ containing a direct summand isomorphic to $\left.Z\left[J^{-1}\right]\right)$, then $A / / G$ is the cokernel of the canonical homomorphism $\operatorname{Hom}\left(Z\left[J^{-1}\right], A\right) \rightarrow A$. Thus $A / / G$ is the image of the Ext-completion map $A \rightarrow \prod_{p \in J} \operatorname{Ext}\left(Z_{p^{\infty}}, G\right)$ of [9].

Theorem 5.2. For an abelian group $G$ and $n \geq 2$, let $W$ be the Moore space $M(G, n)$ whose only reduced homology group is $G$ in dimension $n$, and let $J$ be the set of all primes $p$ with $p: G \cong G$. Then, for each space $Y \in H o_{*}$,

$$
\pi_{i} P_{W} Y \cong \begin{cases}\pi_{i} Y & \text { for } i<n \\ \pi_{i} Y / / G & \text { for } i=n \\ \pi_{i} Y \otimes Z_{(J)} & \text { for } i>n \text { when } G \text { is torsion. }\end{cases}
$$

Moreover, there is a natural short exact sequence

$$
0 \rightarrow \prod_{p \in J} \operatorname{Ext}\left(Z_{p^{\infty}}, \pi_{i} Y\right) \rightarrow \pi_{i} P_{W} Y \rightarrow \prod_{p \in J} \operatorname{Hom}\left(Z_{p^{\infty}}, \pi_{i-1} Y\right) \rightarrow 0
$$

for $i>n$ when $G$ is not torsion.

This theorem may be applied repeatedly to determine $\pi_{*} P_{W} Y$ when $W$ is an arbitrary wedge of Moore spaces. For instance

Theorem 5.3. Let $W=M\left(G_{1}, n_{1}\right) \vee M\left(G_{2}, n_{2}\right)$ with $2 \leq n_{1}<n_{2}$. Then for $W_{1}=M\left(G_{1}, n_{1}\right)$ and $W_{2}=M\left(G_{1} \oplus G_{2}, n_{2}\right)$, there is a natural equivalence $P_{W} Y \simeq P_{W_{2}}\left(P_{W_{1}} Y\right)$ for $Y \in H o_{*}$.

We devote the rest of this section to proving the above theorems. Clearly

Lemma 5.4. For an abelian group $G$ and $n \geq 2$, a connected space $Y \in H o_{*}$ is $M(G, n)$-periodic if and only if $\operatorname{Hom}\left(G, \pi_{i} Y\right)=0=\operatorname{Ext}\left(G, \pi_{i} Y\right)$ for each $i>n$ and $\operatorname{Hom}\left(G, \pi_{n} Y\right)=0$.

For an abelian group $A$ and set $J$ of primes, the condition that $\operatorname{Hom}\left(Z\left[J^{-1}\right], A\right)=0=\operatorname{Ext}\left(Z\left[J^{-1}\right], A\right)$ is equivalent to the condition that 
$\operatorname{Hom}(Q, A)=0=\operatorname{Ext}(Q, A)$ with $\operatorname{Ext}\left(Z_{p^{\infty}}, A\right)=0$ for each prime $p \notin J$. Such an $A$ is called $J$-cotorsion and decomposes naturally as a product

$$
A \cong \prod_{p \in J} \operatorname{Ext}\left(Z_{p^{\infty}}, A\right)
$$

of $p$-cotorsion (also called Ext- $p$-complete) factors for $p \in J$ as in [9]. For a given abelian group $G$, the class of all abelian groups $X$ with $\operatorname{Hom}(G, X)=$ $0=\operatorname{Ext}(G, X)$ will be denoted by $C(G)$, and the set of all primes $p$ such that $p: G \cong G$ will be denoted by $J_{G}$.

Lemma 5.5. For an abelian group $G$, the class $C(G)$ consists of the $J_{G}$-local abelian groups when $G$ is torsion, and consists of the $J_{G}$-cotorsion abelian groups when $G$ is not torsion.

Proof. Let $C(G)^{\perp}$ consist of all abelian groups $X$ with $\operatorname{Hom}(X, A)=0=$ $\operatorname{Ext}(X, A)$ for each $A \in C(G)$. Then $C(G)^{\perp}$ is one of the "special classes" determined explicitly in [1]. The result follows since $C(G)$ can be recovered from $C(G)^{\perp}$ as the class of all $Y$ with $\operatorname{Hom}(B, Y)=0=\operatorname{Ext}(B, Y)$ for each $B \in C(G)^{\perp}$.

Using the $E_{*}$-localization $X \rightarrow X_{E}$ in the sense of [2], we have

Proposition 5.6. If $W$ is an $E_{*}$-acyclic space for a spectrum $E$, then each $E_{*}$ local space in $\mathrm{Ho}_{*}$ is $W$-periodic, and each $P_{W}$-equivalence in $\mathrm{Ho}_{*}$ is an $E_{*}$ equivalence. Thus there is a natural $E_{*}$-localization map $\alpha: P_{W} X^{*} \rightarrow X_{E}$ for $X \in H o_{*}$.

5.7. Proof of Theorem 5.2. We may assume by 4.4 that $Y$ is $(n-1)$-connected. The isomorphism $\pi_{n} P_{W} Y \cong \pi_{n} Y / / G$ follows since $\operatorname{Hom}\left(G, \pi_{n} P_{W} Y\right)=0$ by 5.4 and since

$$
u^{*}: \operatorname{Hom}\left(\pi_{n} P_{W} Y, B\right) \cong \operatorname{Hom}\left(\pi_{n} Y, B\right)
$$

whenever $\operatorname{Hom}(G, B)=0$, because $K(B, n)$ is then $W$-periodic. Let $R=$ $Z_{(J)}$ when $G$ is torsion, and let $R=\bigoplus_{p \in J} Z / p$ otherwise. Since $W$ is $H_{*}(; R)$ acyclic, there is a natural $H_{*}(; R)$-localization map $\alpha: P_{W} Y \rightarrow Y_{R}$ by 5.6, where $Y_{R}$ is the $H_{*}(; R)$-localization of $Y$. By 5.4 and $5.5, \pi_{i} P_{W} Y$ is: (a) $J$-local for $i>n$ when $G$ is torsion; (b) $J$-cotorsion for $i>n$ when $G$ is not torsion. Moreover, the maximal divisible subgroup $D$ of $\pi_{n} P_{W} Y \cong$ $\pi_{n} Y / / G$ satisfies $\operatorname{Hom}(G, D)=0$, and thus $D=0$ when $G$ is not torsion. Hence, by [2], $\alpha_{*}: \pi_{i} P_{W} Y \cong \pi_{i} Y_{R}$ for $i>n$, and the theorem follows from our knowledge of $\pi_{i} Y_{R}$.

5.8. Proof of Theorem 5.3. Since the periodization maps $Y \rightarrow P_{W_{1}} Y \rightarrow$ $P_{W_{2}}\left(P_{W_{1}} Y\right)$ are clearly $P_{W}$-equivalences, it suffices to show that $P_{W_{2}}\left(P_{W_{1}} Y\right)$ is $W_{1}$-periodic. This follows by 5.2 using

Lemma 5.9. If $A$ is a $J$-cotorsion abelian group for a set $J$ of primes, and if $G$ is not torsion, then $A / / G$ is $J$-cotorsion.

Proof. Since $A / / G$ is a quotient of $A, \operatorname{Ext}(Q, A / / G)=0$ and $\operatorname{Ext}\left(Z_{p^{\infty}}, A / / G\right)$ $=0$ for each prime $p \notin J$. Since $G$ is not torsion, the maximal divisible subgroup of $A / / G$ is zero and $\operatorname{Hom}(Q, A / / G)=0$. Thus $A / / G$ is $J$-cotorsion. 


\section{CONVERGENT FUnCTORS, $\Gamma$-SPACES, AND MAPPING SPACES}

In this section, we make preparations for a comparison of $P_{\Sigma^{2} W} Y$ with $P_{\Sigma W} Y$ in Section 7. We first discuss Segal's theory of $\Gamma$-spaces [37] from the viewpoint of Bousfield-Friedlander [8].

6.1. Convergent functors and $\Gamma$-spaces. A convergent functor consists of a functor $T:$ Sets $_{*} \rightarrow$ Spaces $_{*}$ from pointed sets to pointed spaces (i.e., pointed simplicial sets) such that $T(*)=*$ and $T$ preserves direct limits over directed systems. A $\Gamma$-space consists of a functor $A: \Gamma^{\circ} \rightarrow$ Spaces $_{*}$ with $A\left(0^{+}\right)=*$, where $\Gamma^{\circ} \subset$ Sets $_{*}$ is the full subcategory of objects $n^{+}=\{0,1, \ldots, n\}$ with basepoint $0 \in n^{+}$for $n \geq 0$. Each $\Gamma$-space $A: \Gamma^{\circ} \rightarrow$ Spaces $_{*}$ extends to a convergent functor $A:$ Sets $_{*} \rightarrow$ Spaces $_{*}$, unique up to natural equivalence, and we identify $\Gamma$-spaces with convergent functors. A $\Gamma$-space is called special when

$$
A\left(p_{1}\right) \times \cdots \times A\left(p_{n}\right): A\left(n^{+}\right) \rightarrow A\left(1^{+}\right) \times \cdots \times A\left(1^{+}\right)
$$

is a weak equivalence for each $n \geq 1$, where $p_{i}: n^{+} \rightarrow 1^{+}$is defined by $p_{i}(i)=1$ and $p_{i}(j)=0$ for $j \neq i$. This is equivalent to the condition that

$$
A\left(E_{1} \vee E_{2}\right) \rightarrow A\left(E_{1}\right) \times A\left(E_{2}\right)
$$

is a weak equivalence for each $E_{1}, E_{2} \in \operatorname{Sets}_{*}$. When $A$ is special, $A\left(1^{+}\right)$has an induced abelian monoid structure in $\mathrm{Ho}_{*}$. A $\Gamma$-space $A$ is called very special when it is special and the abelian monoid $\pi_{0} A\left(1^{+}\right)$is an abelian group. Finally, each $\Gamma$-space $A$ determines a functor $A$ : Spaces $_{*} \rightarrow$ Spaces $_{*}$ with $A X=$ $\operatorname{diag}\left(A X_{\bullet}\right)$. and each very special $\Gamma$-space $A$ determines a reduced homology theory $\pi_{*} A X$.

Example 6.2. For an abelian monoid $M$, there is a convergent functor

$$
\begin{aligned}
& \widetilde{M}: \text { Sets }_{*} \rightarrow \text { Sets }_{*} \subset \text { Spaces }_{*}, \\
& \widetilde{M}(E)=\left(\bigoplus_{x \in E} M x\right) / M *,
\end{aligned}
$$

where each $M x$ is a copy of $M$. Let $M \rightarrow M_{g r p}$ be the group completion of $M$, and note that $\widetilde{M}$ is special while $\widetilde{M}_{g r p}$ is very special. There is a natural isomorphism

$$
\pi_{*}\left(\widetilde{M}_{g r p} X\right) \cong \widetilde{H}_{*}\left(X ; M_{g r p}\right)
$$

for $X \in$ Spaces $_{*}$, and Spanier [38, Corollary 5.7] showed that $\widetilde{M} X \rightarrow \widetilde{M}_{g r p} X$ is a weak equivalence when $X$ is connected.

Example 6.3. For $X, Y \in$ Spaces $_{*}$, there is a $\Gamma$-space $\Phi(X, Y)$ with

$$
\Phi(X, Y)\left(n^{+}\right)=\operatorname{map}_{*}\left(X^{n^{+}}, Y\right)
$$

for $n \geq 0$ where $X^{n^{+}}=\operatorname{map}_{*}\left(n^{+}, X\right)=X \times \cdots \times X$ is the product of $n$ copies of $X$.

For $\Gamma$-spaces $A$ and $B$, let $\operatorname{map}_{*}(A, B)$ be the pointed space whose $n$ simplicies are the $\Gamma$-space maps $\left(\Delta^{n} \cup *\right) \wedge A \rightarrow B$ for $n \geq 0$. 
Proposition 6.4. For a convergent functor (i.e., $\Gamma$-space) $A$ and pointed spaces $X$ and $Y$, there is a natural isomorphism

$$
\operatorname{map}_{*}(A X, Y) \cong \operatorname{map}_{*}(A, \Phi(X, Y))
$$

Proof. This is an easy consequence of the natural coend isomorphism

$$
\int^{n^{+}} X^{n^{+}} \wedge A\left(n^{+}\right) \cong A(X)
$$

which follows from the elementary case where $X$ is a pointed set.

Corollary 6.5. Let $X$ be a pointed space and $Y$ be a pointed connected fibrant space. If $\operatorname{map}_{*}(X, Y) \simeq *$, then $\operatorname{map}_{*}(A X, Y) \simeq *$ for each convergent functor A.

Proof. Using the "strict" homotopy theory of $\Gamma$-spaces [8], choose a weak equivalence $B \rightarrow A$ with $B$ cofibrant. This induces a weak equivalence $B X \rightarrow A X$ by [8, Theorem B.2] and it suffices to show $\operatorname{map}_{*}(B X, Y) \simeq *$. The $\Gamma$-space $\Phi(X, Y)$ is fibrant since $Y$ is fibrant, and $\Phi(X, \stackrel{Y}{Y}) \rightarrow *$ is a weak equivalence since $\operatorname{map}_{*}\left(X^{n^{+}}, Y\right) \simeq *$ for each $n \geq 0$. Thus $\operatorname{map}_{*}(B X, Y) \simeq *$ by 6.4 .

We wish to study $\operatorname{map}_{*}(A X, Y)$ when $\operatorname{map}_{*}(X, Y)$ is homotopically discrete, i.e., when $\operatorname{map}_{*}(X, Y) \rightarrow \pi_{0} \operatorname{map}_{*}(X, Y)$ is a weak equivalence.

Lemma 6.6. Let $J, X$, and $Y$ be pointed connected spaces such that $Y$ is fibrant and $\operatorname{map}_{*}(X, Y)$ is homotopically discrete. If $J$ is simply connected, or if $\pi_{1} Y$ acts trivially on $[X, Y]$, then the inclusion $J \vee X \subset J \times X$ induces an equivalence

$$
\operatorname{map}_{*}(J \times X, Y) \simeq \operatorname{map}_{*}(J, Y) \times \operatorname{map}_{*}(X, Y) .
$$

Proof. By 2.1 it suffices to show that $J \vee X \subset J \times X$ induces an equivalence

$$
\operatorname{map}(J \times X, Y) \simeq \operatorname{map}(J \vee X, Y),
$$

or equivalently that it induces a homotopy fiber square:

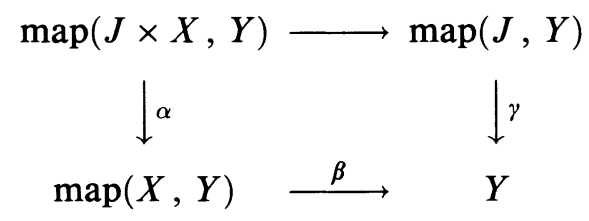

Thus, it suffices to show, for each choice of basepoint $f \in \operatorname{map}(X, Y)$ with $f(*)=*$, that the map of fibers

$$
\alpha^{-1}(f)=\operatorname{map}_{*}(J, \operatorname{map}(X, Y)) \stackrel{\beta_{*}}{\longrightarrow} \operatorname{map}_{*}(J, Y)=\gamma^{-1}(*)
$$

is a weak equivalence. This follows easily because $\beta: \operatorname{map}(X, Y) \rightarrow Y$ has homotopically discrete fiber $\operatorname{map}_{*}(X, Y)$.

A $\Gamma$-space $A$ is called homotopically discrete when $A\left(n^{+}\right)$is homotopically discrete for $n \geq 0$. 
Proposition 6.7. Let $X$ and $Y$ be pointed connected spaces such that $Y$ is fibrant and $\operatorname{map}_{*}(X, Y)$ is homotopically discrete. If $X$ is simply connected or if $\pi_{1} Y$ acts trivially on $[X, Y]$, then $\Phi(X, Y)$ is a homotopically discrete, very special, fibrant $\Gamma$-space, and $[X, Y]$ has a natural abelian group structure.

Proof. By 6.6, $\Phi(X, Y)$ is homotopically discrete, special, and fibrant. Thus $\pi_{0} \Phi(X, Y)\left(1^{+}\right) \cong[X, Y]$ is an abelian monoid, and there is a weak equivalence $\Phi(X, Y) \rightarrow[X, Y]^{\sim}$. Using 6.4 as in the proof of 6.5 , we obtain

$$
\pi_{0} \operatorname{map}_{*}(\widetilde{M} X, Y) \cong \operatorname{Hom}_{\mathscr{M}}(M,[X, Y])
$$

for each $M$ in the category $\mathscr{M}$ of abelian monoids. This combines with the weak equivalence $\widetilde{M} X \rightarrow \widetilde{M}_{g r p} X$ of 6.2 , to give

$$
\operatorname{Hom}_{\mathscr{M}}\left(M_{g r p},[X, Y]\right) \cong \operatorname{Hom}_{\mathscr{M}}(M,[X, Y]) \text {. }
$$

Consequently, $[X, Y]$ is an abelian group and $\Phi(X, Y)$ is very special.

Theorem 6.8. Under the hypotheses of 6.7, if $A$ is a convergent functor, then $\operatorname{map}_{*}(A X, Y)$ is homotopically discrete, and there is a natural isomorphism

$$
[A X, Y] \cong \operatorname{Hom}\left(\pi_{0} A S,[X, Y]\right)
$$

of abelian groups where $S$ is the sphere spectrum.

Proof. As in the proof of 6.5, we may assume that $A$ is cofibrant. Since $\Phi(X, Y)$ is very special and fibrant,

$$
\operatorname{map}_{*}(A X, Y) \cong \operatorname{map}_{*}(A, \Phi(X, Y)) \simeq \operatorname{map}_{*}(A S, \Phi(X, Y) S)
$$

by [8]. The weak equivalence $\Phi(X, Y) \rightarrow[X, Y]^{\sim}$ induces an equivalence of $\Phi(X, Y) S$ with the Eilenberg-Mac Lane spectrum $H[X, Y]$. Thus

$$
\operatorname{map}_{*}(A X, Y) \simeq \operatorname{map}_{*}(A S, H[X, Y])
$$

and the theorem follows.

The following corollary is a version of the "key lemma" initially used by the author to prove the main results of this paper. It involves the symmetric products

$$
X=S P^{1} X \subset S P^{2} X \subset \cdots \subset S P^{\infty} X
$$

of a pointed space $X$.

Corollary 6.9. Under the hypotheses of 6.7, the natural inclusions $X \subset S P^{k} X$ and $X \subset \widetilde{Z} X$ induce equivalences

$$
\operatorname{map}_{*}\left(S P^{k} X, Y\right) \simeq \operatorname{map}_{*}(X, Y) \simeq \operatorname{map}_{*}(\tilde{Z} X, Y)
$$

for $1 \leq k \leq \infty$.

Proof. This follows from 6.8 since the inclusions induce isomorphisms

$$
\pi_{0} S P^{k} S \cong \pi_{0} S \cong \pi_{0} \tilde{Z} S \cong Z
$$




\section{THE TOWER OF SPACES $P_{\Sigma^{i} W} Y$}

For $W, Y \in H o_{*}$, there is a unique $W$-periodization map $\sigma: P_{\Sigma W} Y \rightarrow P_{W} Y$ under $Y$, and thus there is a natural tower

$$
P_{W} Y \stackrel{\sigma}{\longleftarrow} P_{\Sigma W} Y \stackrel{\sigma}{\longleftarrow} P_{\Sigma^{2} W} Y \stackrel{\sigma}{\longleftarrow} \cdots
$$

under $Y$. This reduces to the Postnikov tower when $W=S^{0}$, and we shall show much more generally that the homotopy fibers are successive EilenbergMac Lane spaces. For this we need

7.1. A homological condition. For $n \geq 1$ and a set $J$ of primes, we say that a space $W \in H o_{*}$ satisfies the $n$-supported J-torsion (resp. J-local) condition when:

(i) $\widetilde{H}_{*}(W ; Z)$ is $J$-torsion (resp. $J$-local);

(ii) $\widetilde{H}_{i}(W ; Z)=0$ for $i<n$;

(iii) $H^{n}(W ; Z / p) \neq 0$ for each $p \in J\left(\right.$ resp. $\left.H^{n}\left(W ; Z_{(J)}\right) \neq 0\right)$.

We devote most of this section to proving

Theorem 7.2. For $W \in H o_{*}$ satisfying the $n$-supported J-torsion (resp. J-local) condition, for $Y \in H o_{*}$, and for $i \geq 1$, the homotopy fiber of the map $\sigma$ : $P_{\Sigma^{i+1} W} Y \rightarrow P_{\Sigma^{i} W} Y$ is an Eilenberg-Mac Lane space $K\left(G_{i}, n+i\right)$ for some $J$ torsion (resp. $J$-local) abelian group $G_{i}$.

Of course, it suffices to assume $i=1$. For later use, we give another version of this theorem with different conditions on $W$ in 7.7 below, covering the remaining cases where $\widetilde{H}_{*}(W ; Z)$ is $p$-torsion. In subsequent work, Dror Farjoun and Smith [15] have proved a generalization showing that the homotopy fiber of $\sigma: P_{\Sigma^{2} W} Y \rightarrow P_{\Sigma W} Y$ is a (possibly infinite) product of EilenbergMac Lane spaces when $W$ is an arbitrary connected space. We have used some of their ideas to simplify our original proof. Theorems 7.2 and 7.7 extend easily to cover $\sigma: P_{\Sigma W} Y \rightarrow P_{W} Y$ when $Y \in H o_{*}$ is an $H$-space. However, for a space $W$ with $\widetilde{H}_{*}(W ; Z)=0$, the homotopy fiber of $\sigma: P_{\Sigma W} W \rightarrow P_{W} W$ is equivalent to $W$ by 2.3 . Finally, 7.2 and 2.9 combine to give

Corollary 7.3. For $W \in H o_{*}$ satisfying the n-supported J-torsion (resp. J-local) condition, for $Y \in \mathrm{Ho}_{*}$, and for $i \geq 1$, the maps

$$
Y \stackrel{u}{\longrightarrow} P_{\Sigma^{i} W} Y \stackrel{\sigma}{\longrightarrow} P_{\Sigma W} Y
$$

give the $(n+i)$-th Moore-Postnikov factorization of $u: Y \rightarrow P_{\Sigma W} Y$.

Consequently the successive periodizations $u: Y \rightarrow P_{\Sigma^{i} W} Y$ for $i \geq 1$ are easily obtained from $u: Y \rightarrow P_{\Sigma W} Y$.

Our proof of 7.2 will depend on two lemmas. For a set $J$ of primes, a space $X \in H o_{*}$ is called a $J$-torsion (resp. $J$-local) $G E M$ when $X \simeq \prod_{m=1}^{\infty} K\left(G_{m}, m\right.$ ) for a sequence of $J$-torsion (resp. $J$-local) abelian groups $G_{m}$.

Lemma 7.4. Let $W \in H o_{*}$ satisfy the n-supported J-torsion (resp. J-local) condition, and let $Y \in H o_{*}$ be connected with $\operatorname{map}_{*}(W, Y) \simeq *$. If $B \in H o_{*}$ 
is an $(n-1)$-connected J-torsion (resp. J-local) GEM, then $\operatorname{map}_{*}(B, Y) \simeq *$ and $P_{W} B \simeq *$.

Proof. By 6.5, $\operatorname{map}_{*}(W, Y) \simeq *$ implies $\operatorname{map}_{*}(\tilde{Z} W, Y) \simeq *$ and hence $\operatorname{map}_{*}\left(K\left(H_{n} W, n\right), \stackrel{Y}{ }\right) \simeq *$. By 4.6, the class of abelian groups $G$ with $\operatorname{map}_{*}(K(G, n), Y) \simeq *$ is closed under extensions, cokernels, and direct limits over directed systems. We conclude that $\operatorname{map}_{*}(K(G, n), Y) \simeq *$ for each $J$ torsion (resp. $J$-local) abelian group $G$. The lemma now follows by 4.6 and a direct limit argument.

Lemma 7.5. Let $W \in H o_{*}$ satisfy the $n$-supported J-torsion (resp. J-local) condition, and let $X \in H_{*}$. If $P_{W} X \simeq *$, then $\widetilde{Z} X$ is an $(n-1)$-connected $J$-torsion (resp. J-local) GEM.

Proof. Since $P_{W} X \simeq *$, the construction of $X \rightarrow P_{W} X$ in 2.8 shows that $\widetilde{H}_{*}(X ; Z)$ is $J$-torsion (resp. $J$-local) and $(n-1)$-connected. The lemma now follows since $\widetilde{Z} X$ is a simplicial abelian group with $\pi_{*} \widetilde{Z} X \cong \widetilde{H}_{*}(X ; Z)$.

7.6. Proof of Theorem 7.2. The homotopy fiber $F$ of $P_{\Sigma^{2} W} Y \rightarrow P_{\Sigma W} Y$ is $n$ connected by 2.9 and is $\Sigma^{2} W$-periodic with $P_{\Sigma W} F \simeq *$ by 4.2 and 4.3 . Thus $\Omega^{2} F$ is $W$-periodic and $P_{W} \Omega F \simeq *$ by 3.1. Hence, $\operatorname{map}_{*}\left(\Omega F, \Omega^{2} F\right) \simeq *$ and $\operatorname{map}_{*}(\Omega F, \Omega F)$ is homotopically discrete. The "key lemma" 6.9 now shows that $\Omega F$ is a homotopy retract of $\tilde{Z} \Omega F$, and 7.5 shows that $\tilde{Z} \Omega F$ is an $(n-1)$-connected $J$-torsion (resp. $J$-local) GEM. Since $\Omega F$ is $\Sigma W$-periodic, it is also a homotopy retract of $P_{\Sigma W}(\tilde{Z} \Omega F)$, and the theorem now follows since $P_{\Sigma W}(\widetilde{Z} \Omega F)$ is of the form $K(G, n)$ for a $J$-torsion (resp. $J$-local) abelian group $G$ by 2.7 and 7.4.

Finally, we must give a supplemental version of Theorem 7.2. For $n \geq 1$ and a prime $p$, we say that a space $W \in H o_{*}$ satisfies the $n$-supported divisible p-torsion condition when

(i) $\widetilde{H}_{*}(W ; Z)$ is $p$-torsion;

(ii) $\widetilde{H}_{i}(W ; Z)=0$ for $i<n$;

(iii) $H_{n}(W ; Z)$ is a nontrivial divisible $p$-torsion abelian group.

Theorem 7.7. For $W \in H o_{*}$ satisfying the $n$-supported divisible p-torsion condition, for $Y \in H o_{*}$, and for $i \geq 1$, the homotopy fiber of the map $P_{\Sigma^{i+1} W} Y \rightarrow$ $P_{\Sigma^{i} W} Y$ is equivalent to a product $K\left(G_{i}, n+i\right) \times K\left(G_{i}^{\prime}, n+i+1\right)$, where $G_{i}$ is a divisible p-torsion abelian group and $G_{i}^{\prime}$ is a $Z_{p^{\infty}}$-reduced p-torsion abelian group.

Proof. The proof is essentially the same as for 7.2. However, in place of ordinary Eilenberg-Mac Lane spaces, we must use products $K(G, m) \times K\left(G^{\prime}, m+1\right)$ where $G$ is a divisible $p$-torsion abelian group and $G^{\prime}$ is a $Z_{p^{\infty}}$-reduced $p$ torsion abelian group. The homotopy category of such products, for a given $m \geq 1$, is equivalent to the abelian category of Ext- $p$-complete abelian groups. This equivalence may be obtained by using the $p$-completion functor $F_{p^{\infty}}$ of [9] to carry each such product to an Eilenberg-Mac Lane space $K(\bar{G}, m+1)$, where $\bar{G}$ is an Ext- $p$-complete abelian group. Inversely, for each Ext- $p$-complete 
abelian group $\bar{G}$, the "p-torsion part of $K(\bar{G}, m+1)$ ",

$$
\mathrm{t}_{p} K(\bar{G}, m+1)=\operatorname{Fib}(K(\bar{G}, m+1) \rightarrow K(\bar{G}[1 / p], m+1)),
$$

is a product of the above sort (see 14.1).

\section{THE MAIN FIBRATION THEOREM}

Our earlier fibration theorem in 4.1 involved a bothersome mixture of the $W$ periodization and the $\Sigma W$-periodization. We can now give a fibration theorem involving only the $\Sigma W$-periodization.

Theorem 8.1. For $W \in H o_{*}$ satisfying the $n$-supported J-torsion (resp. J-local) condition of 7.1 and for a homotopy fiber sequence $F \rightarrow X \rightarrow B$ of pointed spaces, the map from $P_{\Sigma W} F$ to the homotopy fiber of $P_{\Sigma W} X \rightarrow P_{\Sigma W} B$ has homotopy fiber of the form $K(G, n)$ for a J-torsion (resp. J-local) abelian group $G$. Moreover, the action of $\pi_{1} P_{\Sigma W} F$ on $G$ is trivial.

Proof. We may assume that $B$ is connected and apply 4.1 to give a natural map

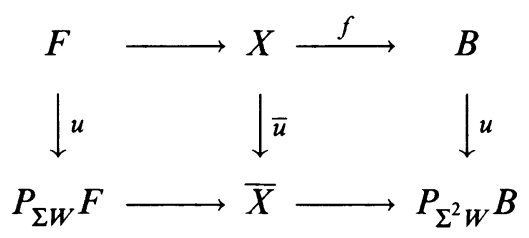

of homotopy fiber sequences, where $\bar{u}$ is a $P_{\Sigma W}$-equivalence and $\bar{X}$ is $\Sigma^{2} W$ periodic. This determines a natural diagram of pointed spaces

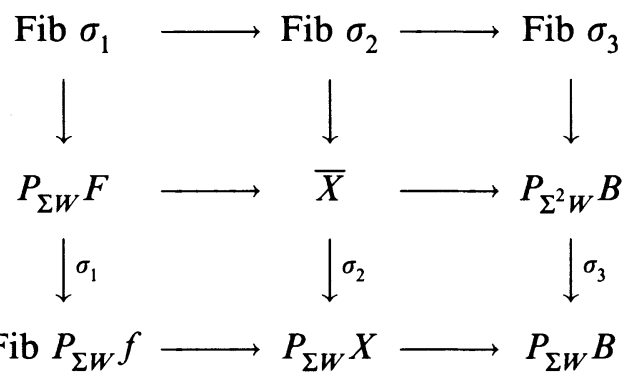

whose rows and columns are homotopy fiber sequences. Theorem 7.2 shows that Fib $\sigma_{2} \simeq K\left(G_{2}, n+1\right)$ and Fib $\sigma_{3} \simeq K\left(G_{3}, n+1\right)$, where $G_{2}$ and $G_{3}$ are $J$-torsion (resp. $J$-local) abelian groups. Thus $\pi_{*}$ Fib $\sigma_{1}$ is also $J$-torsion (resp. $J$-local) abelian and $\pi_{i}$ Fib $\sigma_{1}=0$ for $i \neq n, n+1$. Since Fib $\sigma_{1}$ is $\Sigma W$-periodic by 4.2 , it must have $\pi_{n+1}$ Fib $\sigma_{1}=0$ by 7.4. Thus Fib $\sigma_{1}$ has the required form and $\pi_{1} P_{\Sigma W} F$ acts trivially on $\pi_{n}$ Fib $\sigma_{1}$ since it acts trivially on $\pi_{n+1}$ Fib $\sigma_{3}$.

We may view $K(G, n)$ as an error term measuring the failure of $P_{\Sigma W} F \rightarrow$ $P_{\Sigma W} X \rightarrow P_{\Sigma W} B$ to be a homotopy fiber sequence. When $W$ satisfies the $n$ supported divisible $p$-torsion condition of 7.7, this error term is of the form $K(G, n) \times K\left(G^{\prime}, n+1\right)$ for a divisible $p$-torsion abelian group $G$ and a $Z_{p^{\infty}}$ reduced $p$-torsion abelian group $G^{\prime}$. In subsequent work, Dror Farjoun and 
Smith [15] have proved a generalization showing that the error term is a (possibly infinite) product of Eilenberg-Mac Lane spaces when $W$ is an arbitrary connected space. Theorem 8.1 also generalizes to fiber squares, although the statement on trivial action must be omitted, even when $W=S^{n}$.

Theorem 8.2. For $W \in H o_{*}$ satisfying the $n$-supported J-torsion (resp. J-local) condition and a homotopy fiber square

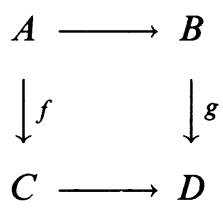

of pointed spaces, the map from $P_{\Sigma W} A$ to the homotopy pull-back of $P_{\Sigma W} B \rightarrow$ $P_{\Sigma W} D \leftarrow P_{\Sigma W} C$ has homotopy fiber of the form $K(G, n)$ for a $J$-torsion (resp. $J$-local) abelian group $G$.

Proof. It suffices to show that the homotopy fiber of $\operatorname{Fib}\left(P_{\Sigma W} f\right) \rightarrow \operatorname{Fib}\left(P_{\Sigma W} g\right)$ has the required form $K(G, n)$. Since Fib $f \simeq$ Fib $g$, this follows by 8.1 and 7.4 .

Theorem 8.1 also leads to a mapping space theorem for $P_{\Sigma W}$.

Theorem 8.3. For $W \in H o_{*}$ satisfying the $n$-supported $J$-torsion (resp. J-local) condition and for connected spaces $X, Y \in H o_{*}$ with $X$ equivalent to a finite $C W$ complex, let $F_{v}$ be the homotopy fiber of the map

$$
P_{\Sigma W} \operatorname{map}_{*}(X, Y) \rightarrow \operatorname{map}_{*}\left(X, P_{\Sigma W} Y\right)
$$

at a vertex $v \in P_{\Sigma W} \operatorname{map}_{*}(X, Y)$. Then:

(i) $\pi_{i} F_{v}=0$ for $i \geq n+1$;

(ii) $\pi_{i} F_{v}$ is a J-torsion (resp. J-local) nilpotent group for $i \geq 1$;

(iii) the action of $\pi_{1} P_{\Sigma W} \operatorname{map}_{*}(X, Y)$ on $\pi_{i} F_{v}$ is nilpotent for each $i \geq 1$.

Proof. The result holds for $X=S^{k}$ with $k \geq 1$ by 3.2 and 7.2. We may assume inductively that $X$ is the homotopy cofiber of a map $\alpha: S^{k} \rightarrow X^{\prime}$ where the result holds for $S^{k}$ and $X^{\prime}$. We then deduce the result for $X$ by applying 8.1 to the homotopy fiber sequence

and using the map

$$
\operatorname{map}_{*}(X, Y) \longrightarrow \operatorname{map}_{*}\left(X^{\prime}, Y\right) \stackrel{\alpha^{*}}{\longrightarrow} \operatorname{map}_{*}\left(S^{k}, Y\right)
$$

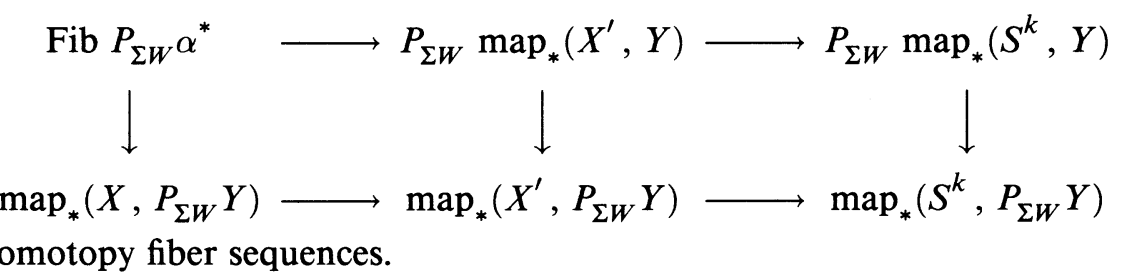

\section{The $P$-Classes of SPACES}

In [4], we introduced equivalence classes of spectra corresponding to the natural equivalence classes of homological localization functors. In almost the 
same way, we now investigate equivalence classes of spaces corresponding to the natural equivalence classes of periodization functors. Dror Farjoun has independently considered these classes. We say that a space $W \in H o_{*}$ is $P$ similar to $W^{\prime} \in H o_{*}$ when the $W$-periodic spaces in $H o_{*}$ are the same as the $W^{\prime}$-periodic spaces. We write $\langle W\rangle$ for the $P$-similarity class, or $P$-class, of $W$. Our main results are classification theorems $(9.12$ and 9.15$)$ for $P$-classes of $p$-torsion suspension spaces.

9.1. The algebra of $P$-classes. For $P$-classes $\langle W\rangle$ and $\left\langle W^{\prime}\right\rangle$, we write $\langle W\rangle \leq$ $\left\langle W^{\prime}\right\rangle$ when each $W^{\prime}$-periodic space is $W$-periodic. This happens if and only if $W \rightarrow *$ is a $P_{W^{\prime}}$-equivalence, which happens if and only if $P_{W^{\prime}}(W) \simeq *$. Moreover, this gives a partial ordering of $P$-classes. Note that $\langle *\rangle \leq\langle W\rangle \leq\left\langle S^{0}\right\rangle$ for each $W \in H o_{*}$. If $\langle W\rangle \leq\left\langle W^{\prime}\right\rangle$, then each $P_{W}$-equivalence in $H o_{*}$ is a $P_{W^{\prime}}$-equivalence, and there is a canonical $W^{\prime}$-localization map $\sigma: P_{W} X \rightarrow$ $P_{W^{\prime}} X$ under $X$. As in [4], there are smash and wedge operations

$$
\begin{aligned}
\langle W\rangle \wedge\left\langle W^{\prime}\right\rangle & =\left\langle W \wedge W^{\prime}\right\rangle, \\
\bigvee_{\alpha}\left\langle W_{\alpha}\right\rangle & =\left\langle\bigvee_{\alpha} W_{\alpha}\right\rangle
\end{aligned}
$$

with the expected properties. For instance, if $\langle V\rangle \leq\left\langle V^{\prime}\right\rangle$ and $\langle W\rangle \leq\left\langle W^{\prime}\right\rangle$, then $\langle V\rangle \wedge\left\langle V^{\prime}\right\rangle \leq\langle W\rangle \wedge\left\langle W^{\prime}\right\rangle$. Thus, if $\langle W\rangle \leq\left\langle W^{\prime}\right\rangle$, then $\left\langle\Sigma^{k} W\right\rangle \leq\left\langle\Sigma^{k} W^{\prime}\right\rangle$ for each $k \geq 0$. Of course, $\langle\Sigma W\rangle \leq\langle W\rangle$ for each $W \in H o_{*}$. Some other elementary general properties of $P$-classes are:

Proposition 9.2. If $A \rightarrow B \rightarrow C$ is a homotopy cofiber sequence, then:

(i) $\langle B\rangle \leq\langle A\rangle \vee\langle C\rangle$;

(ii) $\langle C\rangle \leq\langle B\rangle \vee\langle\Sigma A\rangle \leq\langle B\rangle \vee\langle A\rangle$;

(iii) $\langle\Sigma A\rangle \leq\langle C\rangle \vee\langle\Sigma B\rangle \leq\langle C\rangle \vee\langle B\rangle$.

Proposition 9.3. If $F \rightarrow X \rightarrow B$ is a homotopy fiber sequence with $B$ connected, then:

(i) $\langle B\rangle \leq\langle F\rangle \vee\langle X\rangle$;

(ii) $\langle X\rangle \leq\langle F\rangle \vee\langle B\rangle$;

(iii) $\langle F\rangle \leq\langle X\rangle \vee\langle\Omega B\rangle$.

Proof. Apply 4.8 .

Corollary 9.4. If $B \in H o_{*}$ is connected, then $\langle B\rangle \leq\langle\Omega B\rangle$.

Proposition 9.5. If $F, B \in H o_{*}$, then $\langle F \times B\rangle=\langle F\rangle \vee\langle B\rangle$.

Proof. This follows by 2.7 .

Proposition 9.6. For $V, W \in H o_{*}$ with $V$ connected, the condition $\langle V\rangle \leq$ $\langle\Sigma W\rangle$ holds if and only if $\langle\Omega V\rangle \leq\langle W\rangle$.

Proof. This follows since the condition $P_{\Sigma W} V \simeq *$ is equivalent to $P_{W}(\Omega V) \simeq$ * by 3.1 .

Corollary 9.7. For $k \geq 1$ and for $V, W, W^{\prime} \in H o{ }_{*}$ with $W$ and $W^{\prime}(k-1)$ connected, the following hold:

(i) $\left\langle\Omega^{k} \Sigma^{k} V\right\rangle \leq\langle V\rangle$; 
(ii) $\langle W\rangle \leq\left\langle\Sigma^{k} \Omega^{k} W\right\rangle$;

(iii) if $\langle W\rangle \leq\left\langle W^{\prime}\right\rangle$, then $\left\langle\Omega^{k} W\right\rangle \leq\left\langle\Omega^{k} W^{\prime}\right\rangle$.

Proposition 9.8. If $W \in H o_{*}$ is connected, then $\langle\Sigma \Omega \Sigma W\rangle=\langle\Sigma W\rangle$.

Proof. This follows from Milnor's [28] decomposition of $\Sigma \Omega \Sigma W$.

We next discuss

9.9. The algebra of stable $P$-classes. These classes have also been considered by Dror Farjoun. For each $W \in H o_{*}$, there is a decreasing sequence of $P$ classes

$$
\langle W\rangle \geq\langle\Sigma W\rangle \geq\left\langle\Sigma^{2} W\right\rangle \geq \cdots,
$$

and we say that $W \in H o_{*}$ is stably $P$-similar to $W^{\prime} \in H o_{*}$ when $\left\langle\Sigma^{j} W\right\rangle \leq$ $\left\langle W^{\prime}\right\rangle$ and $\left\langle\Sigma^{k} W^{\prime}\right\rangle \leq\langle W\rangle$ for some $j, k \geq 0$. For this it is sufficient, but not necessary, to have $\left\langle\Sigma^{h} W\right\rangle=\left\langle\Sigma^{i} W^{\prime}\right\rangle$ for some $h, i \geq 0$. For instance, the Moore space wedges $M^{3}(p) \vee M^{4}(q)$ and $M^{3}(p) \vee M^{5}(q)$ are stably $P$-similar for distinct primes $p$ and $q$, although none of their iterated suspensions are $P$-similar. We write $\{W\}$ for the stable $P$-similarity class, or stable $P$-class, of $W$. For stable $P$-classes $\{W\}$ and $\left\{W^{\prime}\right\}$, we write $\{W\} \leq\left\{W^{\prime}\right\}$ when $\left\langle\Sigma^{j} W\right\rangle \leq\left\langle W^{\prime}\right\rangle$ for some $j \geq 0$. This gives a partial ordering of the stable $P$-classes. There are smash and wedge operations

$$
\begin{aligned}
& \{W\} \wedge\left\{W^{\prime}\right\}=\left\{W \wedge W^{\prime}\right\}, \\
& \{W\} \vee\left\{W^{\prime}\right\}=\left\{W \vee W^{\prime}\right\}
\end{aligned}
$$

with the expected properties, but there is not a well-defined infinite wedge for stable $P$-classes. Since $\langle W\rangle \leq\left\langle W^{\prime}\right\rangle$ implies $\{W\} \leq\left\{W^{\prime}\right\}$, and since $\{\Sigma W\}=\{W\}$, the results 9.2, 9.3, 9.4, 9.5, 9.7 (i), 9.7 (ii), 9.8, and the "if" part of 9.6, all still hold when \langle\rangle is replaced by \{\} . For a prime $p$ and $m, n \geq 1$, Ravenel and Wilson [36] showed that $K(Z / p, n)$ is $K(m)_{*}$-acyclic (or equivalently $K(m)^{*}$-acyclic) if and only if $n>m$, where $K(m)$ is the $m$-th Morava $K$-theory spectrum. Thus

$$
\{K(Z / p, 1)\}>\{K(Z / p, 2)\}>\{K(Z / p, 3)\}>\cdots
$$

by 9.4 , and consequently 9.7 (iii) and the "only if" part of 9.6 fail when \langle\rangle is replaced by \{\} .

To go further, we need

Theorem 9.10. For $n \geq 1$ and a set $J$ of primes, if $W \in H o_{*}$ satisfies the $n$-supported $J$-torsion (resp. J-local) condition, and if $k \geq 1$, then

$$
\begin{aligned}
\langle\Sigma W\rangle & =\left\langle\Sigma^{k} W\right\rangle \vee \bigvee_{p \in J}\langle K(Z / p, n+1)\rangle \\
\text { resp. }\langle\Sigma W\rangle & \left.=\left\langle\Sigma^{k} W\right\rangle \vee\left\langle K\left(Z_{(J)}, n+1\right)\right\rangle\right) .
\end{aligned}
$$

Proof. The inequality $\langle\Sigma W\rangle \geq \cdots$ follows by 7.4 . For the inequality $\langle\Sigma W\rangle$ $\leq \cdots$, let $Y \in H o_{*}$ be a connected space which is $\Sigma^{k} W$-periodic and $K(Z / p, n+1)$-periodic for each $p \in J$ (resp. $K\left(Z_{(J)}, n+1\right)$-periodic). Then 
let $F$ be the homotopy fiber of $u: Y \rightarrow P_{\Sigma W} Y$ and apply 7.2 to show that $\pi_{i} F$ is $J$-torsion (resp. $J$-local) for $n+1 \leq i \leq n+k-1$ and $\pi_{i} F=0$ otherwise. When $i \geq n+1, F$ is $K(Z / p, i)$-periodic for each $p \in J$ (resp. $K\left(Z_{(J)}, i\right)$ periodic) by 2.6 and 7.4. Consequently, $\pi_{*} F=0$ and $Y \simeq P_{\Sigma W} Y$. Hence, the inequality $\langle\Sigma W\rangle \leq \cdots$ follows.

Using 7.7 in place of 7.2, we also obtain

Theorem 9.11. For $n \geq 1$ and a prime $p$, if $W \in H o{ }_{*}$ satisfies the n-supported divisible p-torsion condition, and if $k \geq 1$, then

$$
\langle\Sigma W\rangle=\left\langle\Sigma^{k} W\right\rangle \vee\left\langle K\left(Z_{p^{\infty}}, n+1\right)\right\rangle .
$$

We now focus on the $p$-torsion suspension spaces. Let $\operatorname{conn}(X)$ denote the connectivity of $X$, where $X$ is a space or a graded abelian group.

Theorem 9.12. For a prime $p$, let $W$ and $W^{\prime}$ be pointed spaces such that $\widetilde{H}_{*}(W ; Z)$ and $\widetilde{H}_{*}\left(W^{\prime} ; Z\right)$ are p-torsion. Then the following are equivalent:

(i) $\langle\Sigma W\rangle \leq\left\langle\Sigma W^{\prime}\right\rangle$;

(ii) $\{\Sigma W\} \leq\left\{\Sigma W^{\prime}\right\}, \operatorname{conn}(\Sigma W) \geq \operatorname{conn}\left(\Sigma W^{\prime}\right)$, and $\operatorname{conn} \widetilde{H}^{*}(\Sigma W ; Z / p)$ $\geq \operatorname{conn} \widetilde{H}^{*}\left(\Sigma W^{\prime} ; Z / p\right)$.

Proof. Clearly (i) $\Rightarrow$ (ii). Given (ii), we have $\left\langle\Sigma^{k} W\right\rangle \leq\left\langle\Sigma W^{\prime}\right\rangle$ for some $k \geq 1$ and $\langle K(G, n+1)\rangle \leq\left\langle K\left(G^{\prime}, n^{\prime}+1\right)\right\rangle$, where $n=\operatorname{conn}(\Sigma W)$ with

and $n^{\prime}=\operatorname{conn}\left(\Sigma W^{\prime}\right)$ with

$$
G= \begin{cases}Z / p & \text { when } H^{n+1}(\Sigma W ; Z / p) \neq 0, \\ Z_{p^{\infty}} & \text { otherwise }\end{cases}
$$

$$
G^{\prime}= \begin{cases}Z / p & \text { when } H^{n+1}\left(\Sigma W^{\prime} ; Z / p\right) \neq 0 \\ Z_{p^{\infty}} & \text { otherwise. }\end{cases}
$$

We deduce (i) by applying 9.10 and 9.11 to give

$$
\langle\Sigma W\rangle=\left\langle\Sigma^{k} W\right\rangle \vee\langle K(G, n+1)\rangle \leq\left\langle\Sigma W^{\prime}\right\rangle \vee\left\langle K\left(G^{\prime}, n^{\prime}+1\right)\right\rangle=\left\langle\Sigma W^{\prime}\right\rangle .
$$

By Theorem 9.12, the $P$-classification problem for $p$-torsion suspension spaces is effectively reduced to a stable $P$-classification problem. The latter problem seems difficult in general, but has been completely solved for $p$-torsion finite $C W$-complexes using a classification theorem of Devinatz, Hopkins, and Smith, which we now recall in a form suggested by Ravenel [35]. Let $F H o_{(p)} \subset$ $H o_{*}$ be the full subcategory of all $X \in H o_{*}$ such that $\bigoplus_{i=0}^{\infty} \widetilde{H}_{i}(X ; Z)$ is a finitely generated $Z_{(p)}$-module, i.e., $\Sigma^{\infty} X$ is equivalent to a $p$-local finite spectrum. A full subcategory $\mathrm{C} \subset \mathrm{FHo}(p)$ is called thick when it is nonempty and satisfies the conditions:

(i) in a homotopy cofiber sequence $X \stackrel{f}{\longrightarrow} Y \longrightarrow$ Cof $f$, if two of the spaces belong to $C$, then so does the third;

(ii) if a wedge of spaces $X \vee Y$ belongs to $C$, then so do $X$ and $Y$. 
Thus, for any spectrum $E$, the $E_{*}$ - (or $E^{*}$-) acyclic spaces in $F H o_{(p)}$ form a thick subcategory. For $m \geq 0$, let $K(m)$ denote the $m$-th Morava $K$-theory spectrum at the prime $p$. In particular, let $K(0)$ denote the rational spectrum $H Q$. As shown in [33, Theorem 2.11], for each space $X \in F H o_{(p)}$ with $\widetilde{H}_{*}(X ; Z) \neq 0$, there exists an integer $n \geq 0$ such that $\widetilde{K(m)_{*}} X=0$ for all $m<n$ and $\widetilde{K(m)_{*}} X \neq 0$ for all $m \geq n$. This $n$ is called the type of $X$ at the prime $p$, and we write type $(X)=n$. A space $X \in F H o_{(p)}$ with $\widetilde{H}_{*}(X ; Z)=0$ is said to have type $\infty$. As shown by Mitchell [30] and subsequently by Hopkins and Smith [16], [17], for each prime $p$ and $n \geq 0$, there exists a space $X \in F H o(p)$ with type $(X)=n$.

Theorem 9.13 (Thick subcategory theorem). If $C \subset F H o(p)$ is a thick subcategory, then

$$
\text { obj } C=\left\{X \in F H o_{(p)} \mid \operatorname{type}(X) \geq n\right\}
$$

for some $n$ with $0 \leq n \leq \infty$.

This is proved in [16, Theorem 7], [17], [35] using the nilpotence theorem of Devinatz, Hopkins, and Smith [12]. Dror Farjoun has established the following consequence.

Theorem 9.14. For a prime $p$ and spaces $W, W^{\prime} \in F H o_{(p)},\{W\} \leq\left\{W^{\prime}\right\}$ if and only if type $(W) \geq \operatorname{type}\left(W^{\prime}\right)$.

Proof. The "only if" part follows since the conditions $\{W\} \leq\left\{W^{\prime}\right\}$ and $\widetilde{K(m)}{ }^{*} W^{\prime}=0$ imply $\widetilde{K(m)}{ }^{*} W=0$. For the "if" part, let $C \subset F H o_{(p)}$ be the full subcategory of all spaces $X \in F H o_{(p)}$ such that $\{X\} \leq\left\{W^{\prime}\right\}$. Then $C$ is thick by 9.2 , and thus

$$
\text { obj } C=\left\{X \in F H o_{(p)} \mid \operatorname{type}(X) \geq n\right\}
$$

for some $n \geq 0$. Using the already proved "only if" part, we find that $n=$ type $\left(W^{\prime}\right)$, and thus type $(W) \geq \operatorname{type}\left(W^{\prime}\right)$ implies $\{W\} \leq\left\{W^{\prime}\right\}$.

Theorems 9.12 and 9.14 imply the following $P$-classification theorem.

Theorem 9.15. For a prime $p$ and spaces $W, W^{\prime} \in F H_{(p)}$ of type $>0$, the following are equivalent:

(i) $\langle\Sigma W\rangle \leq\left\langle\Sigma W^{\prime}\right\rangle$;

(ii) type $(\Sigma W) \geq \operatorname{type}\left(\Sigma W^{\prime}\right)$ and $\operatorname{conn}(\Sigma W) \geq \operatorname{conn}\left(\Sigma W^{\prime}\right)$.

This theorem shows that $\langle\Sigma W\rangle=\left\langle\Sigma W^{\prime}\right\rangle$ if and only if type $(\Sigma W)=$ type $\left(\Sigma W^{\prime}\right)$ and $\operatorname{conn}(\Sigma W)=\operatorname{conn}\left(\Sigma W^{\prime}\right)$. To be more explicit, we survey

9.16. The $P$-classes of finite $p$-torsion suspension spaces. We work over a fixed prime $p$, and for each $n \geq 0$ we choose a space $\bar{V}_{n} \in F H o_{(p)}$ of type $n+1$ with $\operatorname{conn}\left(\Sigma \bar{V}_{n}\right)$ minimal. Then the possible P-classes of p-torsion suspension spaces in $\mathrm{FHo}_{(p)}$ are precisely the $\left\langle\Sigma^{i} \bar{V}_{n}\right\rangle$ for $i \geq 1$ and $n \geq 0$. We know that 
$\operatorname{conn}\left(\Sigma \bar{V}_{n}\right) \geq n+1$ for each $n \geq 0$, since the condition $\widetilde{K(n)} \bar{V}_{n}=0$ implies $\widetilde{K(n)}{ }^{*} K(Z / p, j)=0$ for $j=\operatorname{conn}\left(\Sigma \bar{V}_{n}\right)$ by 7.4. Moreover,

$$
\operatorname{conn}\left(\Sigma \bar{V}_{n}\right) \leq \operatorname{conn}\left(\Sigma \bar{V}_{n+1}\right)
$$

for each $n \geq 0$, since type $\left(\bar{V}_{n} \vee \bar{V}_{n+1}\right)=n+1$. Clearly, $\operatorname{conn}\left(\Sigma \bar{V}_{0}\right)=1$ since we may let $\bar{V}_{0}=M^{2}(p)=S^{1} \cup_{p} e^{2}$. Also, $\operatorname{conn}\left(\Sigma \bar{V}_{1}\right)=2$ when $p$ is odd since we may let $\bar{V}_{1}$ be the cofiber of the Adams map

$$
A: \Sigma^{2 p-2} M^{3}(p) \rightarrow M^{3}(p)
$$

constructed in [11]. When $p=2$, an Adams map

$$
A: \Sigma^{8} M^{j}(2) \rightarrow M^{j}(2)
$$

is constructed for $j=6$ in [29] and for $j=5$ in [22], [31]. Thus, $2 \leq$ $\operatorname{conn}\left(\Sigma \bar{V}_{1}\right) \leq 4$ when $p=2$. We emphasize that $\bar{V}_{1}$ need not be constructed as the cofiber of an Adams map.

\section{THE $v_{n}$-PERIODIZATION OF SPACES}

We work over a fixed prime $p$. In view of the classification of finite $p$ torsion suspension spaces in 9.16 , it is natural to focus on the $\Sigma \bar{V}_{n}$-periodization for $n \geq 0$. However, we suspect that almost all of the $P$-classes $\left\langle\Sigma \bar{V}_{n}\right\rangle$ are highly iterated suspensions of $P$-classes of nonfinite $p$-torsion spaces, and we incorporate that possibility into our construction of the $v_{n}$-periodization.

10.1. The fundamental $P$-classes $\left\langle\Sigma W_{n}\right\rangle$. For each $n \geq 0$ we choose a space $W_{n} \in H o_{*}$ such that:

(i) the stable $P$-class $\left\{W_{n}\right\}$ equals $\left\{\bar{V}_{n}\right\}$;

(ii) $W_{n}$ satisfies the $k$-supported $p$-torsion condition of 7.1 for some $k \geq 1$;

(iii) the above value of $k$ is the smallest possible for choices of $W_{n}$ satisfying (i) and (ii).

By 9.12 , the $P$-class $\left\langle\Sigma W_{n}\right\rangle$ is uniquely determined by the above conditions, and $\left\langle\Sigma \bar{V}_{n}\right\rangle=\left\langle\Sigma^{j} W_{n}\right\rangle$ for some $j \geq 1$. We know that $\operatorname{conn}\left(\Sigma W_{n}\right) \geq n+1$ for each $n \geq 0$ by the argument of 9.16. Moreover, as we explain in 13.7 and 13.8, we conjecture for $n \geq 0$ that $\operatorname{conn}\left(\Sigma W_{n}\right)=n+1$ and that $W_{n}$ may be chosen as $B \vee K(Z / p, n+1)$ for any space $B \in F H o_{(p)}$ of type $n+1$. This is known for $n=0,1$. In general,

$$
\operatorname{conn}\left(\Sigma W_{n}\right) \leq \operatorname{conn}\left(\Sigma W_{n+1}\right)
$$

for each $n \geq 0$, by the argument of 9.16 , and thus $\left\langle\Sigma W_{n+1}\right\rangle \leq\left\langle\Sigma W_{n}\right\rangle$ by 9.12 . We let

$$
c(n)=\operatorname{conn}\left(\Sigma W_{n}\right)+1 \text {. }
$$

10.2. The $v_{n}$-periodization of spaces. For $n \geq 0$ and $Y \in H o_{*}$, we let $P_{v_{n}} Y$ denote $P_{\Sigma W_{n}} Y$ and call $u: Y \rightarrow P_{v_{n}} Y$ the $v_{n}$-periodization or $v_{n}$-localization of $Y$. We also call a $\Sigma W_{n}$-periodic space $v_{n}$-periodic or $v_{n}$-local and call a 
$P_{\Sigma W_{n}}$-equivalence a $P_{v_{n}}$-equivalence. For any $B \in F H o_{(p)}$ of type $n+1$, we may immediately recover $u: Y \rightarrow P_{\Sigma B} Y$ from $u: Y \rightarrow P_{v_{n}} Y$ by using a MoorePostnikov factorization as in 7.3. Note that the fibration theorems of Section 8 apply to $v_{n}$-periodizations for $n \geq 0$. Thus

Theorem 10.3. For a homotopy fiber sequence $F \rightarrow X \rightarrow B$ of pointed spaces, the map from $P_{v_{n}} F$ to the homotopy fiber of $P_{v_{n}} X \rightarrow P_{v_{n}} B$ has homotopy fiber of the form $K(G, c(n)-1)$ for a p-torsion abelian group $G$.

10.4. The chromatic tower of spaces. Since $\left\langle\Sigma W_{n+1}\right\rangle \leq\left\langle\Sigma W_{n}\right\rangle$ for $n \geq 0$, there is a natural tower

$$
P_{v_{0}} Y \leftarrow P_{v_{1}} Y \leftarrow P_{v_{2}} Y \leftarrow \cdots
$$

under each $Y \in H o_{*}$, and the homotopy inverse limit is equivalent to $Y$ by 2.9 . Like the chromatic tower in stable homotopy theory, this provides successive approximations to $Y$ allowing "successively higher sorts of periodicity" (see 11.9).

The $v_{n}$-periodization is well understood on Postnikov spaces.

Proposition 10.5. For $n \geq 0$ and a connected space $Y \in H o_{*}$ with $\pi_{i} Y=0$ for sufficiently large $i$, there are natural isomorphisms

$$
\pi_{i} P_{v_{n}} Y \simeq \begin{cases}\pi_{i} Y & \text { for } i<c(n), \\ \pi_{i} Y / /(Z / p) & \text { for } i=c(n), \\ \pi_{i} Y \otimes Z[1 / p] & \text { for } i>c(n),\end{cases}
$$

where $\pi_{i} Y / /(Z / p)$ is the quotient of $\pi_{i} Y$ by its $p$-torsion subgroup.

Proof. For $A=M^{c(n)}(p)$, the homotopy fiber, Fib $u$, of $u: Y \rightarrow P_{\Sigma A} Y$ is a $(c(n)-1)$-connected $p$-torsion Postnikov space by 5.2. By 7.4, $P_{v} K(G, j) \simeq *$ for each $p$-torsion abelian group $G$ and $j \geq c(n)$. Thus by $4.8, P_{v_{n}}($ Fib $u) \simeq *$ and $u: Y \rightarrow P_{\Sigma A} Y$ is a $P_{v_{n}}$-equivalence. The result now follows from 5.2 since $P_{\Sigma A} Y$ is $v_{n}$-periodic because $\left\langle\Sigma W_{n}\right\rangle \leq\langle\Sigma A\rangle$ by 9.12 .

\section{ON $v_{n}$-PERIODIZATION OF SPACES AND $v_{m}$-PERIODIC HOMOTOPY GROUPS}

We continue to work over a fixed prime $p$ and proceed to establish close relations between the theory of $v_{n}$-periodization of spaces and that of $v_{m}$ periodic homotopy groups. These groups, especially for $m=i$ and $m=2$, have figured prominently in recent work of Mahowald, Thompson, and others (see [20], [23], [24]).

We begin by recalling convenient versions of the stable nilpotence and periodicity theorems of Devinatz, Hopkins, and Smith [12], [16], [17], [35]. As in 9.13, let $\mathrm{FHo}_{(p)} \subset H o_{*}$ be the full subcategory of all $X \in H o_{*}$ such that $\bigoplus_{i=0}^{\infty} \widetilde{H}_{i}(X ; Z)$ is a finitely generated $Z_{(p)}$-module. Two maps $u, v: X \rightarrow Y$ in $F H o_{(p)}$ are called stably homotopic when $\Sigma^{k} u \simeq \Sigma^{k} v$ for some $k \geq 0$. For a self-map $f: \Sigma^{d} X \rightarrow X$ in $F H o_{(p)}$ and $i>0$, let $f^{i}: \Sigma^{i d} X \rightarrow X$ denote the 
composite $f \circ \Sigma^{d} f \circ \ldots \circ \Sigma^{(i-1) d} f$, and call $f$ stably nilpotent when $f^{i}$ is stably homotopic to the trivial map for some $i>0$. Devinatz, Hopkins, and Smith [12], [16], [35] have proved

Theorem 11.1 (Nilpotence theorem). For a self-map $f: \Sigma^{d} X \rightarrow X$ in $\mathrm{FHo}_{(p)}$ with $d \geq 0$, if $\widetilde{K(m)_{*}} f=0$ for all $m \geq 0$, then $f$ is stably nilpotent.

For a space $X \in \mathrm{FHo}_{(p)}$ of type $n$ with $0<n<\infty$, a map $\omega: \Sigma^{d} X \rightarrow X$ with $d \geq 0$ is called a $v_{n}$ self-map when $\widetilde{K(n)_{*}} \omega$ is an isomorphism and $\widetilde{K(m)_{*}} \omega=0$ for all $m \neq n$. Hopkins and Smith [16], [17], [35] have proved

Theorem 11.2 (Periodicity theorem). Let $X \in F \mathrm{Ho}_{(p)}$ be a space of type $n$ with $0<n<\infty$. Then:

(i) $\Sigma^{k} X$ has a $v_{n}$ self-map for some $k \geq 0$;

(ii) if $\omega: \Sigma^{d} X \rightarrow X$ is a $v_{n}$ self-map of $X$, then $d$ is a positive multiple of $2 p^{n}-2$;

(iii) if $\omega: \Sigma^{d} X \rightarrow X$ and $\tau: \Sigma^{e} X \rightarrow X$ are $v_{n}$ self-maps of $X$, then $\omega^{i}: \Sigma^{i d} X \rightarrow X$ is stably homotopic to $\tau^{j}: \Sigma^{j e} X \rightarrow X$ for some $i, j>0$ with id $=j e$.

11.3. The $v_{m}$-periodic homotopy groups. For each $m \geq 1$, we may choose a pointed space $V_{m-1}$ and a pointed map $\omega: \Sigma^{d} V_{m-1} \rightarrow V_{m-1}$ such that $V_{m-1}$ is equivalent to a finite $C W$-complex of type $m$ in $F H o_{(p)}$ and $\omega$ represents a $v_{m}$ self-map of $V_{m-1}$ in $F H o_{(p)}$.

For a space $Y \in H o_{*}$ and integer $t$, we let $\pi_{t}\left(Y ; V_{m-1}\right)=\left[\Sigma^{t} V_{m-1}, Y\right]$ and define the $v_{m}$-periodic homotopy group $v_{m}^{-1} \pi_{t}\left(Y ; V_{m-1}\right)$ as the colimit of the sequence

$$
\pi_{t}\left(Y ; V_{m-1}\right) \stackrel{\omega^{*}}{\longrightarrow} \pi_{t+d}\left(Y ; V_{m-1}\right) \stackrel{\omega^{*}}{\longrightarrow} \pi_{t+2 d}\left(Y ; V_{m-1}\right) \longrightarrow \cdots .
$$

Although $v_{m}^{-1} \pi_{t}\left(Y ; V_{m-1}\right)$ depends on the choice of $V_{m-1}$, it does not depend on the choice of $\omega$ by 11.2. Note that these groups are periodic with

$$
\omega^{*}: v_{m}^{-1} \pi_{t}\left(Y ; V_{m-1}\right) \cong v_{m}^{-1} \pi_{t+d}\left(Y ; V_{m-1}\right) \text {, }
$$

and that each fiber sequence $F \rightarrow X \rightarrow B$ has a long exact sequence of $v_{m^{-}}$ periodic homotopy groups. Following Mahowald and Thompson [23], for $m \geq$ 1 and a pointed fibrant space $Y$, we construct the homotopy colimit space $T_{m} Y$ of the sequence

$$
\operatorname{map}_{*}\left(V_{m-1}, Y\right) \stackrel{\omega^{*}}{\longrightarrow} \operatorname{map}_{*}\left(\Sigma^{d} V_{m-1}, Y\right) \stackrel{\omega^{*}}{\longrightarrow} \operatorname{map}_{*}\left(\Sigma^{2 d} V_{m-1}, Y\right) \longrightarrow \cdots .
$$

There is a natural isomorphism

$$
\pi_{t} T_{m} Y \cong v_{m}^{-1} \pi_{t}\left(Y ; V_{m-1}\right)
$$

for $t \geq 0$ and a natural equivalence

$$
\omega^{*}: T_{m} Y \simeq \Omega^{d} T_{m} Y
$$

making $T_{m} Y$ an infinite loop space. 
Lemma 11.4. The space $T_{m} Y$ is $v_{n}$-periodic for each $n \geq m$.

Proof. Since $T_{m} Y$ is a periodic infinite loop space and $\left\langle\Sigma V_{n}\right\rangle=\left\langle\Sigma^{j} W_{n}\right\rangle$ for some $j \geq 1$, it suffices to show that $T_{m} Y$ is $\Sigma V_{n}$-periodic for $n \geq m$. Since $\Sigma V_{n} \in H o_{*}$ is equivalent to a finite $C \stackrel{m}{W}$-complex, it now suffices by exponentiation to show that the tower

$$
\Sigma V_{n} \wedge V_{m-1} \longleftarrow \Sigma V_{n} \wedge \Sigma^{d} V_{m-1} \longleftarrow \Sigma V_{n} \wedge \Sigma^{2 d} V_{m-1} \longleftarrow \cdots
$$

is pro-trivial in $H o_{*}$. This follows by observing that $\widetilde{K}(j)_{*}\left(\Sigma V_{n} \wedge \omega\right)=0$ for each $j \geq 0$, and then applying 11.1 to show that

$$
\Sigma V_{n} \wedge \omega: \Sigma V_{n} \wedge \Sigma^{d} V_{m-1} \longrightarrow \Sigma V_{n} \wedge V_{m-1}
$$

is stably nilpotent.

Theorem 11.5. For each space $Y \in H o_{*}$ and each $n \geq m \geq 1$, the $v_{n^{-}}$ periodization map $u: Y \longrightarrow P_{v_{n}} Y$ induces an isomorphism

$$
v_{m}^{-1} \pi_{*}\left(Y ; V_{m-1}\right) \cong v_{m}^{-1} \pi_{*}\left(P_{v_{n}} Y ; V_{m-1}\right) \text {. }
$$

Thus each $P_{v_{n}}$-equivalence in $H o_{*}$ is a $v_{m}^{-1} \pi_{*}\left(-; V_{m-1}\right)$-equivalence for $n \geq$ $m \geq 1$.

Proof. We may assume that $Y$ is connected and fibrant. The diagram

$$
\begin{aligned}
P_{\Sigma V_{n}} \operatorname{map}_{*}\left(V_{m-1}, Y\right) & \rightarrow P_{\Sigma V_{n}} \operatorname{map}_{*}\left(\Sigma^{d} V_{m-1}, Y\right) \\
P_{\Sigma V_{n}} \operatorname{map}_{*}\left(V_{m-1}, P_{\Sigma V_{n}} Y\right) \rightarrow P_{\Sigma V_{n}} \operatorname{map}_{*}\left(\Sigma^{d} V_{m-1}, P_{\Sigma V_{n}} Y\right) & \rightarrow P_{\Sigma V_{n}} \operatorname{map}_{*}\left(\Sigma^{2 d} V_{m-1}, Y\right) \rightarrow \cdots \\
& \rightarrow P_{\Sigma V_{n}} \operatorname{map}_{*}\left(\Sigma^{2 d} V_{m-1}, P_{\Sigma V_{n}} Y\right) \rightarrow \cdots
\end{aligned}
$$

induces a map $T_{m} u: T_{m} Y \rightarrow T_{m} P_{\Sigma V_{n}} Y$ of homotopy colimits by 11.4 and 11.6 below. Since each vertical map is a $\pi_{i}$-equivalence for $i \geq \operatorname{conn}\left(\Sigma V_{n}\right)+2$ by 8.3 , so is $T_{m} u: T_{m} Y \rightarrow T_{m} P_{\Sigma V_{n}} Y$. Thus, by periodicity, we find

$$
v_{m}^{-1} \pi_{*}\left(Y ; V_{m-1}\right) \cong v_{m}^{-1} \pi_{*}\left(P_{\Sigma V_{n}} Y ; V_{m-1}\right) \cong v_{m}^{-1} \pi_{*}\left(P_{\Sigma W_{n}} Y ; V_{m-1}\right) \text {. }
$$

We have used

Lemma 11.6. For a directed system $\left\{X_{\alpha}\right\}$ of pointed spaces and a finite $C W$ complex $A \in H o_{*}$, there is a natural equivalence

$$
P_{A}\left(\operatorname{hocolim} X_{\alpha}\right) \simeq \underset{\alpha}{\operatorname{hocolim}}\left(P_{A} X_{\alpha}\right) \text {. }
$$

Proof. This follows by 2.5 since the right side is clearly $A$-periodic.

Proposition 11.7. For each $Y \in H o_{*}$ and $n \geq 1$, the natural map

$$
\pi_{t}\left(P_{v_{n}} Y ; V_{n-1}\right) \longrightarrow v_{n}^{-1} \pi_{t}\left(P_{v_{n}} Y ; V_{n-1}\right)
$$

is monic when $t=1$ and an isomorphism when $t \geq 2$. 
Proof. The map $\omega: \Sigma^{d} V_{n-1} \rightarrow V_{n-1}$ has homotopy cofiber, Cof $\omega$, of type $\geq n+1$. Thus, for $t \geq 1,\left\langle\Sigma^{t} \operatorname{Cof} \omega\right\rangle \leq\left\langle\Sigma W_{n}\right\rangle$ and $\left[\Sigma^{t} \operatorname{Cof} \omega, P_{v_{n}} Y\right]=0$ as required.

Proposition 11.8. For each space $Y \in H o_{*}$ and $m>n \geq 0$,

$$
\pi_{t}\left(P_{v_{n}} Y ; V_{m-1}\right) \cong v_{m}^{-1} \pi_{t}\left(P_{v_{n}} Y ; V_{m-1}\right) \cong 0
$$

when $t \geq 1$.

Proof. This follows since $\left\langle\Sigma^{t} V_{m-1}\right\rangle \leq\left\langle\Sigma W_{n}\right\rangle$.

11.9. Periodic homotopy groups and the chromatic tower. For $Y \in H o_{*}$, we consider the chromatic tower

$$
P_{v_{0}} Y \longleftarrow P_{v_{1}} Y \longleftarrow P_{v_{2}} Y \longleftarrow \cdots
$$

under $Y$ as in 10.4, and let $\widetilde{P}_{v_{n}} Y$ denote the homotopy fiber of $P_{v_{n}} Y \rightarrow P_{v_{n-1}} Y$. By $11.5,11.7$, and 11.8 , there are natural isomorphisms

$$
\begin{aligned}
& v_{m}^{-1} \pi_{*}\left(P_{v_{n}} Y ; V_{m-1}\right) \cong \begin{cases}v_{m}^{-1} \pi_{*}\left(Y ; V_{m-1}\right) & \text { for } m \leq n, \\
0 & \text { for } m>n,\end{cases} \\
& v_{m}^{-1} \pi_{*}\left(\widetilde{P}_{v_{n}} Y ; V_{m-1}\right) \cong \begin{cases}v_{n}^{-1} \pi_{*}\left(Y ; V_{n-1}\right) & \text { for } m=n, \\
0 & \text { for } m \neq n\end{cases}
\end{aligned}
$$

Moreover, for $t \geq 2$ and $n \geq 1$, there are natural isomorphisms

$$
v_{n}^{-1} \pi_{t}\left(Y ; V_{n-1}\right) \cong \pi_{t}\left(P_{v_{n}} Y ; V_{n-1}\right) \cong \pi_{t}\left(\widetilde{P}_{v_{n}} Y ; V_{n-1}\right)
$$

Thus, $\widetilde{P}_{v_{n}} Y$ captures the groups $v_{n}^{-1} \pi_{t}\left(Y ; V_{n-1}\right)$ in "pure harmonic form", and exposes them as ordinary homotopy groups $\pi_{t}\left(\widetilde{P}_{v_{n}} Y ; V_{n-1}\right)$. We now turn to a study of periodic homotopy equivalences.

Theorem 11.10. For a map $\varphi: X \rightarrow Y$ in $H o_{*}$ and $n \geq 1$, the following are equivalent:

(i) $\varphi_{*}: \pi_{i} P_{v_{n}} X \cong \pi_{i} P_{v_{n}} Y$ for sufficiently large $i$

(ii) $\varphi_{*}: v_{m}^{-1} \pi_{*}\left(X ; V_{m-1}\right) \cong v_{m}^{-1} \pi_{*}\left(Y ; V_{m-1}\right)$ for each $m$ with $1 \leq m \leq n$ and $\varphi_{*}: \pi_{i} X \otimes Z[1 / p] \cong \pi_{i} Y \otimes Z[1 / p]$ for sufficiently large $i$.

Proof. By a homotopy fiber argument using 10.3, it suffices to show, for a connected space $F \in H o_{*}$, the equivalence of:

(i) ${ }^{\prime} \pi_{i} P_{v_{n}} F \cong 0$ for sufficiently large $i$;

(ii) ${ }^{\prime} v_{m}^{-1} \pi_{*}\left(F ; V_{m-1}\right) \cong 0$ for each $m$ with $1 \leq m \leq n$ and $\pi_{i} F \otimes Z[1 / p] \cong$ 0 for sufficiently large $i$. 
Given (i) ${ }^{\prime}$, we deduce that $v_{m}^{-1} \pi_{*}\left(P_{v_{n}} F ; V_{m-1}\right) \cong 0$ for $1 \leq m \leq n$ and that $\pi_{i} P_{v_{0}} F \cong \pi_{i} P_{v_{0}} P_{v_{n}} F \cong 0$ for sufficiently large $i$. This implies (ii) ' by 11.5 and 5.2. Given (ii) ${ }^{\prime}$, we deduce that $P_{v_{n}} F$ is $\Sigma V_{m-1}$-periodic for $1 \leq m \leq n$ since

$$
\pi_{t}\left(P_{v_{m}} F ; V_{m-1}\right) \cong v_{m}^{-1} \pi_{t}\left(P_{v_{m}} F ; V_{m-1}\right) \cong 0
$$

for $t \geq 1$. Hence, $P_{v_{m}} F$ is $\Sigma^{k} W_{m-1}$-periodic for some $k \geq 1$, and $\pi_{i} P_{v_{m}} F \cong$ $\pi_{i} P_{v_{m-1}} F$ for sufficiently large $i$ by 7.2 . Since $\pi_{i} P_{v_{0}} F \cong \pi_{i} F \otimes Z[1 / p] \cong 0$ for sufficiently large $i$, condition (i) ' follows.

Corollary 11.11. For a map $\varphi$ in $\mathrm{Ho}_{*}$ and $n \geq 1$, the following are equivalent:

(i) $\varphi_{*}: \pi_{i} \widetilde{P}_{v_{n}} X \cong \pi_{i} \widetilde{P}_{v_{n}} Y$ for sufficiently large $i$;

(ii) $\varphi_{*}: v_{n}^{-1} \pi_{*}\left(X ; V_{n-1}\right) \cong v_{n}^{-1} \pi_{*}\left(Y ; V_{n-1}\right)$.

Proof. This follows by 11.9 and 11.10 since $\widetilde{P}_{v_{n}} X$ is $v_{n}$-periodic and simply connected with $p$-torsion homotopy.

This shows that the notion of a $v_{n}^{-1} \pi_{*}\left(-; V_{n-1}\right)$-equivalence does not depend on the choice of $V_{n-1}$. Finally, applying the theory of $v_{n}$-periodizations, we obtain some useful general isomorphisms of $v_{m}$-periodic homotopy groups.

Theorem 11.12. Let $A \rightarrow X \rightarrow Y$ be a homotopy cofiber sequence, and let $Z$ be a pointed space. If $\langle A\rangle \leq\left\langle\Sigma W_{n}\right\rangle$ for some $n \geq 1$, then the map $X \rightarrow Y$ and the inclusion $Z \subset Z \vee A$ induce isomorphisms

$$
\begin{aligned}
& v_{m}^{-1} \pi_{*}\left(X ; V_{m-1}\right) \cong v_{m}^{-1} \pi_{*}\left(Y ; V_{m-1}\right), \\
& v_{m}^{-1} \pi_{*}\left(Z ; V_{m-1}\right) \cong v_{m}^{-1} \pi_{*}\left(Z \vee A ; V_{m-1}\right)
\end{aligned}
$$

for each $m$ with $1 \leq m \leq n$.

Proof. Since $\langle A\rangle \leq\left\langle\Sigma W_{n}\right\rangle$, the maps $* \rightarrow A$ and $A \rightarrow *$ are $P_{v_{n}}$-equivalences, and hence so are the maps $X \rightarrow Y$ and $Z \rightarrow Z \vee A$ by 2.5. The result now follows by 11.10 .

This theorem may be used with

Lemma 11.13. For $n \geq 1$, if $B$ is a space of type $\geq n+1$ in $F H o_{(p)}$, then $\langle\Sigma B\rangle \leq\left\langle\Sigma W_{n}\right\rangle$.

Proof. Since type $(\Sigma B) \geq n+1,9.14$ and 10.1 imply that $\{\Sigma B\} \leq\left\{\Sigma \bar{V}_{n}\right\}=$ $\left\{\Sigma W_{n}\right\}$ and

conn $\widetilde{H}^{*}(\Sigma B ; Z / p)=\operatorname{conn}(\Sigma B) \geq \operatorname{conn}\left(\Sigma W_{n}\right)=\operatorname{conn} \widetilde{H}^{*}\left(\Sigma W_{n} ; Z / p\right)$.

The lemma now follows from 9.12.

Corollary 11.14. For $n \geq 1$, if $\varphi: X \rightarrow Y$ is a $K(n)_{*}$-equivalence in $F H o_{(p)}$, then $\Sigma^{2} \varphi: \Sigma^{2} X \rightarrow \Sigma^{2} Y$ is a $v_{m}^{-1} \pi_{*}\left(-; V_{m-1}\right)$-equivalence for each $m$ with $1 \leq m \leq n$.

Proof. Apply 11.13 with $B=\operatorname{Cof} \varphi$, and then apply 11.12 with $A=\Sigma B$. 


\section{HOMOLOGICAL PROPERTIES OF THE $v_{n}$-PERIODIZATION AND A THEOREM OF THOMPSON}

Working over a fixed prime $p$, we now discuss homological properties of the $v_{n}$-periodization and give a homological criterion, based on a theorem of Thompson, for a map to be a $v_{m}^{-1} \pi_{*}\left(-; V_{m-1}\right)$-equivalence. This criterion will involve a homology theory $S(m)_{*}$ which could be replaced by the Morava $K$ theories $K(i)_{*}$ for $0 \leq i \leq m$ if an unstable telescope conjecture stated in 12.3 were confirmed. The results of this section are preparatory to more definitive results in Section 13 on $P_{v_{n}}$-equivalences of highly connected spaces. We start with

Proposition 12.1. For $n \geq 0$, the $v_{n}$-periodization $u: Y \rightarrow P_{v_{n}} Y$ of a space $Y \in H o_{*}$ is both an $E_{*}$-equivalence and $E^{*}$-equivalence for each spectrum $E$ with $\widetilde{E}^{*}\left(V_{n}\right)=0$.

Proof. Since $V_{n}$ is finite, the condition $\widetilde{E}^{*}\left(V_{n}\right)=0$ implies $\widetilde{E}_{*}\left(V_{n}\right)=0$, and this implies $(\widetilde{\nabla E})^{*}\left(V_{n}\right)=0$ by 4.11 . Since $\left\{W_{n}\right\}=\left\{V_{n}\right\}$, we deduce that $\widetilde{E}^{*}\left(W_{n}\right)=0,(\widetilde{\nabla E})^{*}\left(W_{n}\right)=0$, and $\widetilde{E}_{*}\left(W_{n}\right)=0$. The result now follows by the construction of $u: Y \rightarrow P_{\Sigma W_{n}} Y$ in 2.8 .

For $m \leq n$, the $B P$-related spectra $v_{m}^{-1} B P, E(m)$, and $K(m)$ all satisfy the hypotheses of 12.1, and thus the associated (co)homologies are all preserved by $v_{n}$-periodizations and $P_{v_{n}}$-equivalences. We shall need the "strongest" possible example, $S(n)$, of a $p$-local spectrum satisfying the above hypotheses.

12.2. The spectrum $S(n)$. For $n \geq 0$ let $\mathscr{A}\left(V_{n}\right)$ be the class of all spectra $E$ with $\widetilde{E}^{*}\left(V_{n}\right) \cong 0$. As explained more generally in [4] and [5, $\left.\S 3\right]$, each spectrum $X$ has a universal (initial) map $u: X \rightarrow X / / V_{n}$ to a spectrum $X / / V_{n} \in \mathscr{A}\left(V_{n}\right)$. We let $S(n)$ denote $S_{(p)} / / V_{n}$, where $S_{(p)}$ is the $p$-localized sphere spectrum, and recall that $S(n)$ is a commutative ring spectrum whose multiplication gives an equivalence $S(n) \wedge S(n) \simeq S(n)$. Moreover, there is a natural equivalence $S(n) \wedge X \simeq X_{(p)} / / V_{n}$ for an arbitrary spectrum $X$. As noted by Hal Sadofsky, when $X$ is a finite $p$-local spectrum of type $n$, the spectrum $S(n) \wedge X$ may alternatively be obtained as a Ravenel telescope $\widehat{X}$ using the construction in [33], [34], [35]. The stable class $\langle S(n)\rangle$ is the complement of $\left\langle\Sigma^{\infty} V_{n}\right\rangle$ in $\left\langle S_{(p)}\right\rangle$ by [4] and thus satisfies

$$
\langle S(n)\rangle \geq \bigvee_{i=0}^{n}\langle K(i)\rangle=\langle E(n)\rangle=\left\langle v_{n}^{-1} B P\right\rangle
$$

by [33, Theorem 2.1]. Ravenel's telescope conjecture is equivalent to the assertion that

$$
\langle S(n)\rangle=\bigvee_{i=0}^{n}\langle K(i)\rangle
$$

for each $n$. This holds for $n=0,1$, as shown in [5, Corollary 4.9] using work of Mahowald and Miller, but has now been refuted by Ravenel [34] for $n=2$ when $p \geq 5$. However, the following unstable version remains plausible. 
12.3. An unstable telescope conjecture. A space is $S(n)_{*}$-acyclic if and only if it is $K(i)_{*}$-acyclic for each $i$ with $0 \leq i \leq n$.

In view of the $K(i)_{*}$-acyclicity results of [36], a weaker and even more plausible version is

12.4. A weak unstable telescope conjecture. For each $j>n \geq 0, K(Z / p, j)$ is $S(n)_{*}$-acyclic.

We presently know 12.3 and 12.4 only for $n=0,1$. Note that $S(0) \simeq H Q$ and $S(1)$ is the $K_{(p) *}$-localization of the sphere spectrum (see [5], [33]). We now generalize a key result proved by Thompson [39] in the case $m=1$. Our proof is a direct adaptation of his.

Theorem 12.5. For $m \geq 1$ and $k \geq \operatorname{dim} V_{m-1}$, if $\varphi: X \rightarrow Y$ is a map in Ho such that $\Omega^{k} \varphi: \Omega^{k} X \rightarrow \Omega^{k} Y$ is an $S(m)_{*}$-equivalence, then

$$
\varphi_{*}: v_{m}^{-1} \pi_{*}\left(X ; V_{m-1}\right) \cong v_{m}^{-1} \pi_{*}\left(Y ; V_{m-1}\right) .
$$

Proof. We may assume that $X$ and $Y$ are $k$-connected and fibrant. Since $\varphi$ induces an $S(m)_{*}$-equivalence $\Omega^{k} X \rightarrow \Omega^{k} Y$, it must induce an $S(m)_{*}$ equivalence $\operatorname{map}_{*}(F, X) \rightarrow \operatorname{map}_{*}(F, Y)$ for each pointed connected finite space $F$ with $\operatorname{dim} F \leq k$, by an inductive argument using 4.6. Using the functor $T_{m}$ of 11.3 , we obtain a commutative diagram

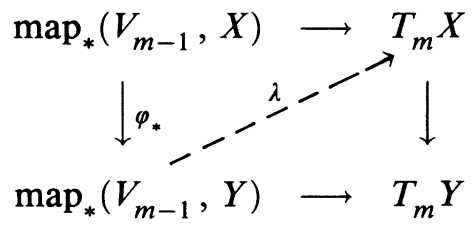

where $\varphi_{*}$ is an $S(m)_{*}$-equivalence, and where the periodic infinite loop spaces $T_{m} X$ and $T_{m} Y$ are $S(m)_{*}$-local by 11.4. Hence, there is a unique map $\lambda$ : $\operatorname{map}_{*}\left(V_{m-1}, Y\right) \rightarrow T_{m} X$ making both triangles commute in $H o_{*}$. The map $\omega: \Sigma^{d} V_{m-1} \rightarrow V_{m-1}$ induces a map from the above solid arrow diagram to its $d$-fold looping, and the resulting square of diagonals

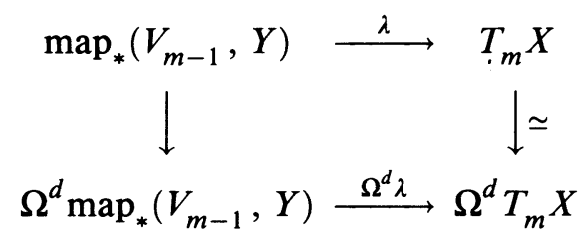

commutes since it is equalized by the $S(m)_{*}$-equivalence

Thus all maps in

$$
\varphi_{*}: \operatorname{map}_{*}\left(V_{m-1}, X\right) \rightarrow \operatorname{map}_{*}\left(V_{m-1}, Y\right) \text {. }
$$

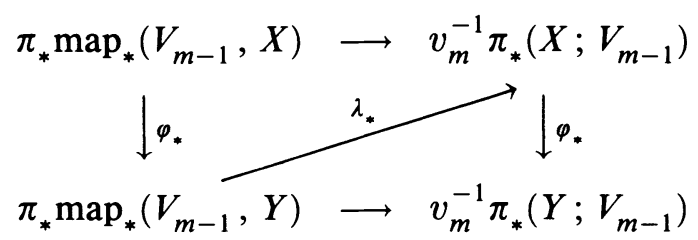


respect the action of $\omega$. Since the horizontal maps are algebraic localizations inverting $\omega, \varphi_{*}: v_{m}^{-1} \pi_{*}\left(X ; V_{m-1}\right) \cong v_{m}^{-1} \pi_{*}\left(Y ; V_{m-1}\right)$ as required.

Note. Each of the spaces $V_{m-1}$, chosen with $\omega: \Sigma^{d} V_{m-1} \rightarrow V_{m-1}$ in 11.3 , is equivalent to a finite $C W$-complex, and $\operatorname{dim} V_{m-1}$ may be interpreted as the minimum possible dimension of such a complex. Thus, we may take $\operatorname{dim} V_{0}=3$ for $p$ odd and $\operatorname{dim} V_{0}=5$ for $p=2$ by 9.16 .

The following corollary was obtained by R. Thompson [39] for $m=1$.

Corollary 12.6. For $m \geq 1$ and $k \geq \operatorname{dim} V_{m-1}$, if $\varphi: X \rightarrow Y$ is an $S(m)_{*}$ equivalence of pointed connected spaces, then

$$
\left(\Sigma^{k} \varphi\right)_{*}: v_{m}^{-1} \pi_{*}\left(\Sigma^{k} X ; V_{m-1}\right) \cong v_{m}^{-1} \pi_{*}\left(\Sigma^{k} Y ; V_{m-1}\right) .
$$

Proof. This follows by 12.5 since the functor $\Omega^{k} \Sigma^{k}: H o_{*} \rightarrow H o_{*}$ preserves generalized homology equivalences of connected spaces by the usual approximation theory (see [39]).

\section{3. $P_{v_{n}}$-EQUIVALENCES OF HIGHLY CONNECTED SPACES}

Working over a fixed prime $p$, we shall establish criteria in 13.3 and 13.15 for a map of $c(n)$-connected spaces to be a $P_{v_{n}}$-equivalence, where $c(n)=$ $\operatorname{conn}\left(\Sigma W_{n}\right)+1$. As explained in 13.8 below, we know that $c(n) \geq n+2$ and conjecture that $c(n)=n+2$ for each $n \geq 0$. We first show that $P_{v_{n}}$ commutes with the $c(n)$-connected cover functor.

Proposition 13.1. For $n \geq 0$ and a space $Y \in H o_{*}$, there is a natural equivalence

$$
P_{v_{n}}(Y\langle c(n)\rangle) \simeq\left(P_{v_{n}} Y\right)\langle c(n)\rangle .
$$

Proof. Since $Y\langle c(n)\rangle$ is the homotopy fiber of the natural map from $Y$ to its $c(n)$-Postnikov section, we may apply 10.3 and 10.5 to show that

$$
\pi_{i} P_{v_{n}} Y\langle c(n)\rangle \rightarrow \pi_{i} P_{v_{n}} Y
$$

is an isomorphism for $i \geq c(n)+1$. The result now follows since $P_{v_{n}}(Y\langle c(n)\rangle)$ is $c(n)$-connected by 2.9 .

13.2. The $v_{n}$-periodization in $H o_{c(n)}$. For $n \geq 0$, let $H o_{c(n)}$ be the full subcategory of $\mathrm{Ho}_{*}$ given by the $c(n)$-connected spaces, and note that $P_{v_{n}}$ restricts to a functor $P_{v_{n}}: H o_{c(n)} \rightarrow H o_{c(n)}$. For a map $\varphi: X \rightarrow Y$ of pointed $c(n)$ connected spaces, let $\widetilde{F}$ ib $\varphi$ denote the $c(n)$-connected cover of the homotopy fiber Fib $\varphi$. By 10.3 and 13.1, there is a natural equivalence

$$
P_{v_{n}}(\tilde{\mathrm{Fib}} \varphi) \simeq \widetilde{\mathrm{Fib}}\left(P_{v_{n}} \varphi\right)
$$

for $n \geq 0$, and thus $P_{v_{n}}$ preserves "fibrations in the sense of $c(n)$-connected homotopy theory". In particular, for $Y \in H o_{c(n)}$, there is a natural equivalence

$$
P_{v_{n}}(\tilde{\Omega} Y) \simeq \widetilde{\Omega}\left(P_{v_{n}} Y\right)
$$


where $\widetilde{\Omega}: H o_{c(n)} \rightarrow H o_{c(n)}$ is the functor with $\widetilde{\Omega} X$ given by the $c(n)$ connected cover of $\Omega X$. Thus, if $\varphi: X \rightarrow Y$ is a $P_{v_{n}}$-equivalence in $H o_{c(n)}$, then so is $\widetilde{\Omega}^{k} \varphi: \widetilde{\Omega}^{k} X \rightarrow \widetilde{\Omega}^{k} Y$ for each $k \geq 0$. Our first characterization of the $P_{v_{n}}$-equivalences in $H o_{c(n)}$ is

Theorem 13.3. For $n \geq 0$, the following conditions on a map $\varphi: X \rightarrow Y$ in $H o_{c(n)}$ are equivalent:

(i) $\varphi$ is a $P_{v_{n}}$-equivalence;

(ii) $\varphi_{*}: v_{m}^{-1} \pi_{*}\left(X ; V_{m-1}\right) \cong v_{m}^{-1} \pi_{*}\left(Y ; V_{m-1}\right)$ for each $m$ with $1 \leq m \leq n$ and $\varphi_{*}: \pi_{*} X \otimes Z[1 / p] \cong \pi_{*} Y \otimes Z[1 / p]$.

Proof. Since $X$ and $Y$ are simply connected, the condition $\varphi_{*}: \pi_{*} X \otimes Z[1 / p]$ $\cong \pi_{*} Y \otimes Z[1 / p]$ is equivalent to $\varphi_{*}: H_{*}(X ; Z[1 / p]) \cong H_{*}\left(Y^{*} ; Z[1 / p]\right)$. Thus (i) $\Rightarrow$ (ii) by 5.6 and 11.5. Given (ii), we deduce from 2.9 and 11.10 that the map $P_{v_{n}} \varphi: P_{v_{n}} X \rightarrow P_{v_{n}} Y$ has homotopy fiber $\operatorname{Fib}\left(P_{v_{n}} \varphi\right)$ such that $\pi_{i} \operatorname{Fib}\left(P_{v_{n}} \varphi\right)$ is: trivial for $i<c(n)^{n}$, trivial for sufficiently large $i$, and $p$-torsion for all $i$. Since $\operatorname{Fib}\left(P_{v_{n}} \varphi\right)$ is $v_{n}$-periodic, we may now apply 10.5 to deduce

$$
\operatorname{Fib}\left(P_{v_{n}} \varphi\right) \simeq P_{v_{n}} \operatorname{Fib}\left(P_{v_{n}} \varphi\right) \simeq *
$$

and thus to deduce (i).

This theorem combines with 13.1 and 10.5 to show

Corollary 13.4. For $n \geq 0$ and a space $Y \in H o_{*}$, the condition $P_{v_{n}} Y \simeq *$ is equivalent to the combined conditions:

(i) $\pi_{i} Y=0$ for $i<c(n)$;

(ii) $\pi_{*} Y$ is p-torsion;

(iii) $v_{m}^{-1} \pi_{*}\left(Y ; V_{m-1}\right)=0$ for each $m$ with $1 \leq m \leq n$.

Using Theorem 13.3, we may also reformulate Thompson's result for suspensions (12.6) as

Theorem 13.5. For $n \geq 0$ and $k \geq \max \left\{2, \operatorname{dim} V_{0}, \ldots, \operatorname{dim} V_{n-1}\right\}$, if $f$ : $X \rightarrow Y$ is an $S(n)_{*}$-equivalence of pointed connected spaces, then $\left(\Sigma^{k} f\right)_{(p)}$ : $\left(\Sigma^{k} X\right)_{(p)} \rightarrow\left(\Sigma^{k} Y\right)_{(p)}$ is a $P_{v_{n}}$-equivalence in $H o_{c(n)}$.

Proof. Since $f$ is an $S(n)_{*}$-equivalence, it is an $S(m)_{*}$-equivalence for $m \leq n$. Thus $f$ induces isomorphisms $\pi_{*}\left(\Sigma^{k} X\right) \otimes Q \cong \pi_{*}\left(\Sigma^{k} Y\right) \otimes Q$ and

$$
v_{m}^{-1} \pi_{*}\left(\Sigma^{k} X ; V_{m-1}\right) \cong v_{m}^{-1} \pi_{*}\left(\Sigma^{k} Y ; V_{m-1}\right)
$$

for $1 \leq m \leq n$ by 12.6. Using the homotopy cofiber Cof $\omega$ of $\omega: \Sigma^{d} V_{n-1} \rightarrow$ $V_{n-1}$, we find

$$
\begin{aligned}
k & \geq \operatorname{dim} V_{n-1} \geq \operatorname{conn}\left(\Sigma V_{n-1}\right)+1=\operatorname{conn}(\Sigma \operatorname{Cof} \omega)+1 \\
& \geq \operatorname{conn}\left(\Sigma W_{n}\right)+1=c(n)
\end{aligned}
$$


for $n \geq 1$. Thus, for $n \geq 0, \Sigma^{k} X$ and $\Sigma^{k} Y$ are in $H o_{c(n)}$, and the result follows from 13.3.

This leads to a homological characterization of the stable $P$-class $\left\{V_{n}\right\}$.

Proposition 13.6. For $n \geq 0$ and $X \in \mathrm{Ho}_{*}$, the following are equivalent:

(i) $\{X\} \leq\left\{V_{n}\right\}$;

(ii) $X$ is $S(n)_{*}$-acyclic and $\widetilde{H}_{*}(X ; Z)$ is p-local.

Proof. Since $\widetilde{S}(n)_{*} V_{n}=0$ and $\widetilde{H}_{*}\left(V_{n} ; Z / q\right)=0$ for each prime $q \neq p$, (i) implies (ii) as in the proof of 12.1. Given (ii), 13.5 implies $P_{v_{n}}\left(\Sigma^{k} X\right) \simeq *$ for $k \geq \max \left\{2, \operatorname{dim} V_{0}, \ldots, \operatorname{dim} V_{n-1}\right\}$. Thus $\left\langle\Sigma^{k} X\right\rangle \leq\left\langle\Sigma W_{n}\right\rangle$ and $\{X\} \leq$ $\left\{W_{n}\right\}=\left\{V_{n}\right\}$.

We now obtain a homological characterization of the integer $c(n)=$ $\operatorname{conn}\left(\Sigma W_{n}\right)+1$.

Proposition 13.7. For $n \geq 0$, let $c_{n}$ be the integer determined by the conditions that $\widetilde{S(n)_{*}} K(Z / p, j) \neq 0$ for $j<c_{n}-1$ and $\widetilde{S(n)_{*}} K(Z / p, j)=0$ for $j \geq$ $c_{n}-1$. Then $c(n)=c_{n}$ and we may choose $W_{n}=B \vee K\left(Z / p, c_{n}-1\right)$ for any space $\mathrm{B} \in \mathrm{FHo} o_{(p)}$ of type $n+1$.

Proof. The space $W_{n}$ chosen in 10.1 must be $S(n)_{*}$-acyclic by the proof of 12.1 , and thus $K(Z / p, c(n)-1)$ is also $S(n)_{*}$-acyclic by 7.4 and 4.11. Hence, $c(n) \geq c_{n}$. By 13.6 and 9.14,

$$
\left\{K\left(Z / p, c_{n}-1\right)\right\} \leq\left\{V_{n}\right\}=\{B\}=\left\{\bar{V}_{n}\right\}
$$

and thus

$$
\left\{B \vee K\left(Z / p, c_{n}-1\right)\right\}=\left\{\bar{V}_{n}\right\} .
$$

This implies that $c_{n} \geq c(n)$ and the proposition follows easily.

13.8. The value of $c(n)$. The weak unstable telescope conjecture of 12.4 now implies that $c(n)=c_{n}=n+2$ for all $n \geq 0$. Since this conjecture is known for $n=0,1$, we conclude that $c(0)=2$ and $c(1)=3$. We also know that $c(n) \geq n+2$ since $\widetilde{K(n)_{*}} K(Z / p, n) \neq 0$ for $n \geq 0$.

13.9. The integer $e(n)$. Although $\widetilde{S(n)})_{*} K(Z / p, c(n)-2) \neq 0$ for $n \geq 0$, it is not evident that $\widetilde{S(n)_{*}} K\left(Z_{p^{\infty}}, c(n)-2\right) \neq 0$. We let $e(n)=0$ when this holds and let $e(n)=1$ when $\widetilde{S(n)_{*}} K\left(Z_{p^{\infty}}, c(n)-2\right)=0$. Since

$$
\widetilde{K(n)_{*}} K\left(Z_{p^{\infty}}, n\right) \cong \widetilde{K(n)_{*}} K(Z, n+1) \neq 0,
$$

we know that $\widetilde{S(n)_{*}} K\left(Z_{p^{\infty}}, n\right) \neq 0$. Thus the weak unstable telescope conjecture implies that $e(n)=0$ for all $n$, and we know that $e(0)=0$ and $e(1)=0$. We also know that the condition $\widetilde{S(n)_{*}} K\left(Z_{p^{\infty}}, j\right)=0$ implies $\widetilde{S(n)}{ }_{*} K(Z / p, j+e(n))=0$. 
Theorem 13.10. For $n \geq 0$ and $k \geq 1+e(n)$, if $X \in H o_{*}$ is an $S(n)_{*}$-acyclic space, then $P_{v_{n}}\left(\Sigma^{k} X\right)_{(p)} \simeq *$ or equivalently $\left\langle\left(\Sigma^{k} X\right)_{(p)}\right\rangle \leq\left\langle\Sigma W_{n}\right\rangle$.

Proof. The result is clear for $n=0$ since $(\Sigma X)_{(p)}$ is a 1-connected $p$-torsion

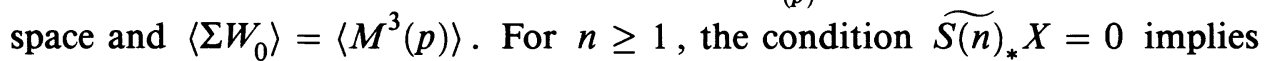
that $\widetilde{H}_{*}(X ; Q)=0$, and thus $\widetilde{H}_{*}\left(X ; Z_{(p)}\right)$ is $p$-torsion. Let $\widetilde{H}_{m}\left(X ; Z_{(p)}\right)$ be the first nontrivial $Z_{(p)}$-homology group of $X$, let $G=Z_{p^{\infty}}$ when $\widetilde{H}_{m}\left(X ; Z_{(p)}\right)$ is $p$-divisible, and let $G=Z / p$ otherwise. Then $\tilde{Z}_{(p)} X$ and $K(G, m)$ are $S(n)_{*}$-acyclic by $6.5,4.11$, and 4.6. Hence $K(Z / p, m+e(n))$ is also $S(n)_{*}$ acyclic and $m+e(n) \geq c(n)-1$ by 13.7. In particular, $m \geq 2$ and $\widetilde{H}_{1}\left(X ; Z_{(p)}\right)$ $=0$. Using the $p$-localization $X_{(p)}=\left(Z_{(p)}\right)_{\infty} X$ of [9], we find that $X \rightarrow X_{(p)}$ is an $H_{*}\left(-; Z_{(p)}\right)$-equivalence and $X_{(p)}$ is an $(m-1)$-connected $p$-torsion space with $\widetilde{H}_{*}\left(X_{(p)} ; Z\right) \cong \widetilde{H}_{*}\left(X ; Z_{(p)}\right)$. Since $\left(\Sigma^{i} X\right)_{(p)} \simeq \Sigma^{i}\left(X_{(p)}\right)$ for $i \geq 0$, we may apply 9.10 and 9.11 to show

$$
\left\langle\left(\Sigma^{k} X\right)_{(p)}\right\rangle=\left\langle\left(\Sigma^{j+k} X\right)_{(p)}\right\rangle \vee\langle K(G, m+k)\rangle
$$

for $j \geq 0$. The theorem now follows since $P_{v_{n}}\left(\Sigma^{j+k} X\right)_{(p)} \simeq *$ for sufficiently large $j$ by 13.5 and $P_{v_{n}} K(G, m+k) \simeq *$ by 10.5 since $m+k \geq m+1+e(n) \geq$ $c(n)$.

We can now prove a strengthened version of Thompson's result for suspensions $(12.6,13.5)$.

Corollary 13.11. For $n \geq 0$ and $k \geq 2+e(n)$, if $\varphi: X \rightarrow Y$ is an $S(n)_{*}$ equivalence of pointed spaces, then $\left(\Sigma^{k} \varphi\right)_{(p)}:\left(\Sigma^{k} X\right)_{(p)} \rightarrow\left(\Sigma^{k} Y\right)_{(p)}$ is a $P_{v_{n}}{ }^{-}$ equivalence.

Proof. Since Cof $\varphi$ is $S(n)_{*}$-acyclic, $P_{v_{n}}\left(\Sigma^{k-1} \operatorname{Cof} \varphi\right)_{(p)} \simeq *$ by 13.10 , and the theorem follows by 2.5 .

Corollary 13.12. For $n \geq 0$ and $k \geq 2+e(n)$, if $Y \in H o_{*}$ is $v_{n}$-periodic with $\pi_{i} Y$ p-local for $i \geq k+1$, then $\Omega^{k} Y$ is $S(n)_{*}$-local.

Proof. This follows from 13.11 by adjunction.

Theorem 13.13. For $n \geq 0$ and $k \geq 1+e(n)$, if $X \in H o_{*}$ is a k-connected p-local space with $\widetilde{S(n)_{*}}\left(\Omega^{k} X\right) \cong 0$, then $P_{v_{n}} X \simeq *$.

Proof. This follows since

$$
\langle X\rangle \leq\left\langle\Sigma^{k} \Omega^{k} X\right\rangle=\left\langle\left(\Sigma^{k} \Omega^{k} X\right)_{(p)}\right\rangle \leq\left\langle\Sigma W_{n}\right\rangle
$$

by 9.7 and 13.10 .

A strengthened version of Thompson's result for loop spaces (12.5) is

Corollary 13.14. For $n \geq 0$ and $k \geq 2+e(n)$, if $\varphi: X \rightarrow Y$ is a map of pointed $k$-connected spaces such that $\Omega^{k} \varphi: \Omega^{k} X \rightarrow \Omega^{k} Y$ is an $S(n)_{*}$-equivalence and $\varphi_{*}: \pi_{*} X \otimes Z[1 / p] \cong \pi_{*} Y \otimes Z[1 / p]$, then $\varphi: X \rightarrow Y$ is a $P_{v_{n}}$-equivalence. 
Proof. $P_{v_{n}}($ Fib $\varphi) \simeq *$ by 4.6 and 13.13. Thus $\varphi$ is a $P_{v_{n}}$-equivalence by 4.8 .

Finally, we shall give a homological characterization of $P_{v_{n}}$-equivalences in $H o_{c(n)}$ supplementing our homotopical characterization in 13.3. Using the $c(n)$-connected loop functor $\widetilde{\Omega}: H o_{c(n)} \rightarrow H o_{c(n)}$ of 13.2, we say that a map $\varphi: X \rightarrow Y$ in $H o_{c(n)}$ is a durable $E_{*^{-}}$(or $E^{*}$-) equivalence for a spectrum $E$ when $\widetilde{\Omega}^{k} \varphi: \widetilde{\Omega}^{k} X \rightarrow \widetilde{\Omega}^{k} Y$ is an $E_{*}-\left(\right.$ or $E^{*}$-) equivalence for each $k \geq 0$.

Theorem 13.15. For $n \geq 0$, the following conditions on a map $\varphi: X \rightarrow Y$ in $H o_{c(n)}$ are equivalent:

(i) $\varphi$ is a $P_{v_{n}}$-equivalence;

(ii) $\varphi$ is a durable $E_{*}$ - (and $E^{*}$-) equivalence for each spectrum $E$ with $\widetilde{E}^{*}\left(V_{n}\right)=0$

(iii) $\varphi$ is a durable $S(n)_{*}$-equivalence and $\varphi_{*}: \pi_{*} X \otimes Z[1 / p] \cong \pi_{*} Y \otimes$ $Z[1 / p]$;

(iv) $\widetilde{\Omega}^{k} \varphi$ is an $S(n)_{*}$-equivalence for some $k \geq 2+e(n)$ and $\varphi_{*}: \pi_{*} X \otimes$ $Z[1 / p] \cong \pi_{*} Y \otimes Z[1 / p]$.

Proof. If (i), then $\widetilde{\Omega}^{k} \varphi$ is a $P_{v_{n}}$-equivalence for each $k \geq 0$ and (ii) follows by 12.1. If (ii), then (iii) follows since $\widetilde{S}(n)^{*} V_{n} \cong 0$ and $\widetilde{H}^{*}\left(V_{n} ; Z[1 / p]\right) \cong 0$. If (iii), then (iv) follows immediately. If (iv), then $\varphi\langle c(n)+k\rangle$ is a $P_{v_{n}}$-equivalence by 13.14 , and (i) follows by 13.3 .

14. ON $v_{1}^{-1} \pi_{*}(-; Z / p)$-EQUIVALENCES AND $K_{*}(-; Z / p)$-EQUIVALENCES

In this final section, we use the preceding results to compare $v_{1}^{-1} \pi_{*}(-; Z / p)$ equivalences and $K_{*}(-; Z / p)$-equivalences. As an application, we confirm the conjecture that the Snaith map

$$
s: \Omega_{0}^{2 n+1} S^{2 n+1} \rightarrow Q\left(\mathbb{R} P^{2 n}\right)
$$

is a $K_{*}(-; Z / 2)$-equivalence. We continue to work over a fixed prime $p$.

Let $M^{n}(p)$ denote the $Z / p$-Moore space $S^{n-1} \cup_{p} e^{n}$, and let $q=2 p-2$ for $p$ odd and $q=8$ for $p=2$. As in 9.16, choose Adams maps (i.e., $K_{*}$ equivalences)

$$
A: M^{j+q}(p) \rightarrow M^{j}(p)
$$

for $j \geq 3$ when $p$ is odd and $j \geq 5$ when $p=2$, where the maps for successive $j$ are obtained by suspension. For a space $X \in H o_{*}$, there is an induced operation

$$
A: \pi_{j}(X ; Z / p) \rightarrow \pi_{j+q}(X ; Z / p)
$$

on the mod- $p$ homotopy groups

$$
\pi_{j}(X ; Z / p)=\left[M^{j}(p), X\right] .
$$


As in 11.3 , we define the $v_{1}$-periodic homotopy groups $v_{1}^{-1} \pi_{*}(X ; Z / p)$ by inverting the action of $A$ on $\pi_{*}(X ; Z / p)$. The $v_{1}$-periodization functor $P_{v_{1}}=$ $P_{\Sigma W_{1}}: H o_{*} \rightarrow H o_{*}$ may be defined using

$$
W_{1}=M^{3}(p) \cup_{A} C M^{3+q}(p)
$$

for $p$ odd and

$$
W_{1}=K(Z / 2,2) \vee\left(M^{5}(2) \bigcup_{A} C M^{5+q}(2)\right)
$$

for $p=2$ by 13.7 .

Since the $S(1)_{*}$-equivalences in $H o_{*}$ are the same as the $K_{*}\left(-; Z_{(p)}\right)$ equivalences, and since $c(1)=3$ and $e(1)=0$, the homological results of Section 13 can immediately be reformulated in terms of $K_{*}\left(-; Z_{(p)}\right)$. However, it is convenient to have the corresponding results for $K_{*}(-; Z / p)$. For these and for future reference we need

14.1. The $p$-cocompletion of a nilpotent space. For a nilpotent group $G$, let tors $_{p} G \subset G$ denote the $p$-torsion subgroup. Let $N H o_{*} \subset \mathrm{Ho}_{*}$ denote the full subcategory of all nilpotent spaces $X \in H o_{*}$ such that $\pi_{1} X /$ tors $_{p} \pi_{1} X$ is uniquely $p$-divisible.

For $X \in N H o_{*}$, we let $\eta: t_{p} X \rightarrow X$ denote the homotopy fiber of the localization $X \rightarrow X[1 / p]$ and call $\eta: t_{p} X \rightarrow X$ the $p$-cocompletion (or $p$ torsion part) of $X$. Note that $t_{p} X$ is a $p$-torsion nilpotent space, and $\eta_{*}$ : $H_{*}\left(t_{p} X ; Z / p\right) \cong H_{*}(X ; Z / p)$. In general, if $A$ is a pointed space with $p$ torsion homology $\widetilde{H}_{*}(A ; Z)$ and if $f: Y \rightarrow Y^{\prime}$ is an $H_{*}(-; Z / p)$-equivalence of pointed nilpotent spaces, then $\operatorname{map}_{*}(A, Y) \simeq \operatorname{map}_{*}\left(A, Y^{\prime}\right)$ and $[A, Y] \cong$ $\left[A, Y^{\prime}\right]$ since $F_{p^{\infty}} f: F_{p^{\infty}} Y \simeq F_{p^{\infty}} Y^{\prime}$ and $\operatorname{map}_{*}(A, Y) \simeq \operatorname{map}_{*}\left(A, F_{p^{\infty}} Y\right)$ by [25, Theorem 1.5]. Thus, the map $\eta: t_{p} X \rightarrow X$ in $N H o_{*}$ is characterized, up to equivalence, by the conditions that $t_{p} X$ is a $p$-torsion nilpotent space and $\eta_{*}: H_{*}\left(t_{p} X ; Z / p\right) \cong H_{*}(X ; Z / p)$. We remark that the functor $t_{p}: N H o_{*} \rightarrow$ $N \mathrm{Ho}_{*}$ is left adjoint to $F_{p^{\infty}}: \mathrm{NHo}_{*} \rightarrow \mathrm{NHo}_{*}$, and these functors restrict to equivalences between the full subcategories of $p$-torsion spaces and of $p$ complete spaces in $\mathrm{NHo}_{*}$. The functor $t_{p}: \mathrm{NHo}_{*} \rightarrow \mathrm{NHo}_{*}$ has the advantage of respecting cofibrations as well as fibrations. A homotopy cofibration $A \rightarrow$ $B \rightarrow C$ in $N H o_{*}$ with $\pi_{1} t_{p} B=0$ is carried to a homotopy cofibration $t_{p} A \rightarrow$ $t_{p} B \rightarrow t_{p} C$, and there is a natural equivalence $t_{p}(\Sigma A) \simeq \Sigma\left(t_{p} A\right)$ for all $A \in$ $\mathrm{NHo}_{*}$. A homotopy fibration $\mathrm{X} \rightarrow \mathrm{Y} \rightarrow \mathrm{Z}$ in $N H o_{*}$ is carried to a homotopy fibration $t_{p} X \rightarrow t_{p} Y \rightarrow t_{p} Z$, and there is a natural equivalence $t_{p}(\Omega Z) \simeq$ $\Omega\left(t_{p} Z\right)$ for all simply connected $Z \in H o_{*}$ with $\pi_{2} Z /$ tors $_{p} \pi_{2} Z$ uniquely $p$ divisible.

Lemma 14.2. For a space $X \in N H o_{*}$, the p-cocompletion $\eta: t_{p} X \rightarrow X$ induces isomorphisms

$$
\begin{aligned}
\eta_{*}: v_{1}^{-1} \pi_{*}\left(t_{p} X ; Z / p\right) & \cong v_{1}^{-1} \pi_{*}(X ; Z / p) \\
\eta_{*}: K_{*}\left(t_{p} X ; Z / p\right) & \cong K_{*}(X ; Z / p)
\end{aligned}
$$


Proof. This follows since $v_{1}^{-1} \pi_{*}(X[1 / p] ; Z / p)=0$ and $\eta_{*}: H_{*}\left(t_{p} X ; Z / p\right) \cong$ $H_{*}(X ; Z / p)$.

The following theorem and its three corollaries extend results of Thompson [39].

Theorem 14.3. For $k \geq 1$, if $X \in H o_{*}$ is a $k$-connected space with

$$
\widetilde{K}_{*}\left(\Omega^{k} X ; Z / p\right)=0,
$$

then $v_{1}^{-1} \pi_{*}(X ; Z / p)=0$.

Proof. Since $\Omega^{k} X$ is $K_{*}(-; Z / p)$-acyclic and $K\left(Z_{p}^{\wedge}, 2\right)$ is $K_{*}(-; Z / p)$-local by [29], we have $H^{i}\left(\Omega^{k} X ; Z_{p}^{\wedge}\right)=0$ for $i=0,1$. Thus

$$
\operatorname{Ext}\left(\pi_{k+1} X, Z_{p}^{\wedge}\right)=0=\operatorname{Hom}\left(\pi_{k+1} X, Z_{p}^{\wedge}\right)
$$

and $\pi_{k+1} X$ is uniquely $p$-divisible by [1]. Thus $t_{p} X$ is a $k$-connected $p$-torsion space with

$$
\widetilde{K}_{*}\left(\Omega^{k} t_{p} X ; Z_{(p)}\right) \cong \widetilde{K}_{*}\left(t_{p} \Omega^{k} X ; X_{(p)}\right)=0 ;
$$

we have $P_{v_{1}}\left(t_{p} X\right) \simeq *$ by 13.13 and

$$
v_{1}^{-1} \pi_{*}(X ; Z / p) \cong v_{1}^{-1} \pi_{*}\left(t_{p} X ; Z / p\right)=0
$$

by 11.5 .

Corollary 14.4. For $k \geq 2$, if $\varphi: X \rightarrow Y$ is a map of pointed $k$-connected spaces such that $\Omega^{k} \varphi$ is a $K_{*}(-; Z / p)$-equivalence, then

$$
\varphi_{*}: v_{1}^{-1} \pi_{*}(X ; Z / p) \cong v_{1}^{-1} \pi_{*}(Y ; Z / p) \text {. }
$$

Proof. Since the homotopy fiber $\operatorname{Fib} \varphi$ is a $(k-1)$-connected space with $\widetilde{K}_{*}\left(\Omega^{k-1}\right.$ Fib $\left.\varphi ; Z / p\right)=0$, we have $v_{1}^{-1} \pi_{*}($ Fib $\varphi ; Z / p)=0$ by 14.3 .

Corollary 14.5. For $k \geq 1$, if $X \in \mathrm{Ho}_{*}$ is a pointed connected space with $\widetilde{K}_{*}(X ; Z / p)=0$, then $v_{1}^{-1} \pi_{*}\left(\Sigma^{k} X ; Z / p\right)=0$.

Proof. This follows from 14.3 since $K_{*}\left(\Omega^{k} \Sigma^{k} X ; Z / p\right)=0$ as in 12.6.

Corollary 14.6. For $k \geq 2$, if $\varphi: X \rightarrow Y$ is a $K_{*}(-; Z / p)$-equivalence of pointed connected spaces, then $\varphi_{*}: v_{1}^{-1} \pi_{*}\left(\Sigma^{k} X ; Z / p\right) \cong v_{1}^{-1} \pi_{*}\left(\Sigma^{k} Y ; Z / p\right)$.

Proof. This follows from 14.4 since $\Omega^{k} \Sigma^{k} \varphi$ is a $K_{*}(-; Z / p)$-equivalence as in 12.6.

Our main result relating $v_{1}^{-1} \pi_{*}(-; Z / p)$-equivalences and $K_{*}(-; Z / p)$ equivalences is

Theorem 14.7. The following conditions on a map $\varphi: X \rightarrow Y$ in $\mathrm{Ho}_{3}$ are equivalent:

(i) $\varphi_{*}: v_{1}^{-1} \pi_{*}(X ; Z / p) \cong v_{1}^{-1} \pi_{*}(Y ; Z / p)$;

(ii) $\varphi$ is a durable $K_{*}(-; Z / p)$-equivalence;

(iii) $\widetilde{\Omega}^{k} \varphi$ is a $K_{*}(-; Z / p)$-equivalence for some $k \geq 2$. 
Proof. If (i), then (ii) follows since $t_{p}(\varphi\langle 4\rangle)$ is a durable $K_{*}(-; Z / p)$-equivalence in $\mathrm{Ho}_{3}$ by 13.3 and 13.15. If (ii), then (iii) follows immediately. If (iii), then (i) follows by 14.4 since $\Omega^{k}(\varphi\langle 3+k\rangle)$ is a $K_{*}(-; Z / p)$-equivalence.

An old conjecture of Miller and Snaith [26] and Mahowald and Ravenel [21] asserts that the Snaith map

$$
s: \Omega_{0}^{2 n+1} S^{2 n+1} \longrightarrow Q\left(\mathbb{R} P^{2 n}\right)
$$

is a $K_{*}(-; Z / 2)$-equivalence for $n \geq 1$. This conjecture was based on Mahowald's earlier insights and was strongly supported by his proof [20] that the Snaith map is a $v_{1}^{-1} \pi_{*}(-; Z / 2)$-equivalence. However, Mahowald and Thompson obtained a surprising counterexample to this conjecture for $n=1$ in [23] using a theorem of Mayorquin to compute $K_{*}\left(\Omega_{0}^{3} S^{3} ; Z / 2\right)$. When work on the present project showed that Mahowald's $v_{1}^{-1} \pi_{*}(-; Z / p)$-equivalence result does in fact imply the conjecture, the author appealed to Mahowald and Thompson, who resolved the dilemma by finding a crucial error in Mayorquin's proof. This cleared the way for

Theorem 14.8. The Snaith map

$$
s: \Omega_{0}^{2 n+1} S^{2 n+1} \longrightarrow Q\left(\mathbb{R} P^{2 n}\right)
$$

is a $K_{*}(-; Z / 2)$-equivalence for $n \geq 1$.

Proof. Since the map $s\langle 3\rangle$ of 3 -connected covers is a $v_{1}^{-1} \pi_{*}(-; Z / 2)$-equivalence by [20], it is a durable $K_{*}(-; Z / 2)$-equivalence in $\mathrm{Ho}_{3}$ by 14.7. Since $\pi_{3} \Omega_{0}^{2 n+1} S^{2 n+1}$ and $\pi_{3} Q\left(\mathbb{R} P^{2 n}\right)$ are torsion, $s\langle 2\rangle$ is also a $K_{*}(-; Z / 2)$-equivalence. Finally, since

$$
s_{*}: \pi_{i} \Omega_{0}^{2 n+1} S^{2 n+1} \longrightarrow \pi_{i} Q\left(\mathbb{R} P^{2 n}\right)
$$

is an isomorphism for $i \leq 2$, we conclude that $s$ is a $K_{*}(-; Z / 2)$-equivalence.

This theorem implies that $s$ is also an equivalence for $K^{*}(-; Z / 2)$, $K_{*}\left(-; Z_{(2)}\right), K^{*}\left(-; Z_{(2)}\right), K O_{*}\left(-; Z_{(2)}\right), K O^{*}\left(-; Z_{(2)}\right)$, etc. We may use this theorem to determine the hitherto inaccessible $\bmod 2 K$-theory of $\Omega_{0}^{2 n+1} S^{2 n+1}$.

Corollary 14.9. There is an isomorphism

$$
K_{*}\left(\Omega_{0}^{2 n+1} S^{2 n+1} ; Z / 2\right) \cong E\left(y_{1}, \ldots, y_{n}\right) \otimes P\left(z_{1}, \ldots, z_{n}\right)
$$

of $Z / 2$-algebras for $n \geq 1$, with exterior generators $y_{i} \in \widetilde{K}_{1}\left(\Omega_{0}^{2 n+1} S^{2 n+1} ; Z / 2\right)$ and polynomial generators $z_{i} \in \widetilde{K}_{0}\left(\Omega_{0}^{2 n+1} S^{2 n+1} ; Z / 2\right)$.

Proof. Since $s: \Omega_{0}^{2 n+1} S^{2 n+1} \rightarrow Q\left(\mathbb{R} P^{2 n}\right)$ is a $K_{*}(-; Z / 2)$-equivalence and a loop map, this follows from the calculation of $K_{*}\left(Q\left(\mathbb{R} P^{2 n}\right) ; Z / 2\right)$ by Miller and Snaith [27].

In very recent work, Lisa Langsetmo [19] has determined all of the $Z / p$ algebras $K_{*}\left(\Omega^{j} S^{2 n+1} ; Z / p\right)$ for $j<2 n$, using $v_{1}^{-1} \pi_{*}(-; Z / p)$-equivalences derived from the work of Mahowald and Thompson [24], and using $K$-theoretic calculations on the resulting "infinite loop space related" models for $\Omega^{j} S^{2 n+1}$. The preceding theorems should permit other calculations of this sort. 


\section{REFERENCES}

1. A. K. Bousfield, Types of acyclicity, J. Pure Appl. Algebra 4 (1974), 293-298.

2. _ - The localization of spaces with respect to homology, Topology 14 (1975), 133-150.

3. __ Constructions of factorization systems in categories, J. Pure Appl. Algebra 9 (1977), 207-220.

4. __ The Boolean algebra of spectra, Comment. Math. Helv. 54 (1979), 368-377.

5. _ The localization of spectra with respect to homology, Topology 18 (1979), 257-281.

6. _ $\ldots$-localizations and $K$-equivalences of infinite loop spaces, Proc. London Math. Soc. 44 (1982), 291-311.

7. _ The simplicial homotopy theory of iterated loop spaces (in preparation).

8. A. K. Bousfield and E. M. Friedlander, Homotopy theory of $\Gamma$-spaces, spectra, and bisimplicial sets, Lecture Notes in Math., vol. 658, Springer-Verlag, Berlin and New York, 1978, pp. 80-130.

9. A. K. Bousfield and D. M. Kan, Homotopy limits, completions and localizations, Lecture Notes in Math., vol. 304, Springer-Verlag, Berlin and New York, 1972.

10. C. Casacuberta, G. Peschke, and M. Pfenninger, On orthogonal pairs in categories and localizations, London Math. Soc. Lecture Note Ser., vol. 175, Cambridge Univ. Press, Cambridge and New York, 1992, pp. 211-223.

11. F. Cohen and J. Neisendorfer, A note on desuspending the Adams map, Math. Proc. Cambridge Philos. Soc. 99 (1986), 59-64.

12. E. Devinatz, M. J. Hopkins, and J. H. Smith, Nilpotence and stable homotopy, Ann. of Math. (2) 128 (1988), 207-242.

13. E. Dror Farjoun, The localization with respect to a map and $v_{1}$-periodicity, Lecture Notes in Math., vol. 1509, Springer-Verlag, Berlin and New York, 1992, pp. 104-113.

14. E. Dror Farjoun, Localizations, fibrations, and conic structures (to appear).

15. E. Dror Farjoun and J. H. Smith, Homotopy localization nearly preserves fibrations (to appear).

16. M. J. Hopkins, Global methods in homotopy theory, London Math. Soc. Lecture Note Ser., vol. 117, Cambridge Univ. Press, Cambridge and New York, 1987, pp. 73-96.

17. M. J. Hopkins and J. H. Smith, Nilpotence and stable homotopy theory II (to appear).

18. D. M. Kan, On c.s.s. complexes, Amer. J. Math. 79 (1957), 449-476.

19. L. Langsetmo, The $K$-theory localization of loops on an odd sphere and application, Topology 32 (1993), 577-585.

20. M. Mahowald, The image of $J$ in the EHP sequences, Ann. of Math. (2) 116 (1982), 65-112.

21. M. Mahowald and D. Ravenel, Toward a global understanding of stable homotopy groups of spheres, Contemp. Math., vol. 58, part II, Amer. Math. Soc., Providence, RI, 1987, pp. 75-88.

22. M. Mahowald and W. Richter, There exists a $v_{1}^{4}$ Adams map $M_{2 l}^{13} \rightarrow M_{2 l}^{5}$ of James filtration two (to appear).

23. M. Mahowald and $\mathrm{R}$. Thompson, $K$-theory and unstable homotopy groups, Contemp. Math., vol. 96, Amer. Math. Soc., Providence, RI, 1989, pp. 273-279.

24. __ The K-theory localization of an unstable sphere, Topology 31 (1992), 133-141.

25. H. Miller, The Sullivan conjecture on maps from classifying spaces, Ann. of Math. (2) 120 (1984), 39-87.

26. H. Miller and V. Snaith, On the $K$-theory of the Kahn-Priddy map, J. London Math. Soc. 20 (1979), 339-342.

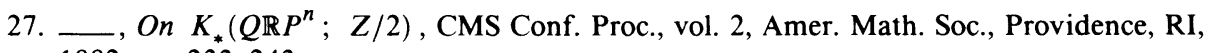
1982, pp. 233-243. 
28. J. Milnor, On the construction FK, London Math. Soc. Lecture Note Ser., vol. 4, Cambridge Univ. Press, Cambridge and New York, 1972, pp. 119-136.

29. G. Mislin, Localizations with respect to K-theory, J. Pure Appl. Algebra 10 (1977), 201-213.

30. S. A. Mitchell, Finite complexes with A(n)-free cohomology, Topology 24 (1985), 227-246.

31. S. Oka, Existence of the unstable Adams map, Mem. Fac. Sci. Kyushu Univ. 42 (1988), 95-108.

32. D. G. Quillen, Homotopical algebra, Lecture Notes in Math., vol. 43, Springer-Verlag, Berlin and New York, 1967.

33. D. C. Ravenel, Localization with respect to certain periodic homology theories, Amer. J. Math. 106 (1984), 351-414.

34. __ Progress report on the telescope conjecture, London Math. Soc. Lecture Note Ser., vol. 176, Cambridge Univ. Press, Cambridge and New York, 1992, pp. 1-21.

35. D. C. Ravenel, Nilpotence and periodicity in stable homotopy theory, Ann. of Math. Stud., no. 128, Princeton Univ. Press, Princeton, NJ, 1992.

36. D. C. Ravenel and W. S. Wilson, The Morava $K$-theories of Eilenberg-Mac Lane spaces and the Conner-Floyd conjecture, Amer. J. Math. 102 (1980), 691-748.

37. G. Segal, Categories and cohomology theories, Topology 13 (1974), 293-312.

38. E. Spanier, Function spaces and duality, Ann. of Math. (2) 69 (1959), 142-198.

39. R. Thompson, $A$ relation between $K$-theory and unstable homotopy groups with an application to $B \Sigma_{p}$, Contemp. Math., vol. 146, Amer. Math. Soc., Providence, RI, 1993, pp. 431-439.

40. Z. Yosimura, Universal coefficient sequences for cohomology theories of $C W$ spectra, Osaka J. Math. 12 (1975), 305-323.

DePartment of Mathematics, Statistics, and Computer SCIENCE, University of Illinois at Chicago, Chicago, Illinois 60607

E-mail address: U22048Quic vm.bitnet 